\title{
Köthe-Bochner Spaces and Some Geometric Properties Related to Rotundity and Smoothness
}

\author{
Jan-David Hardtke \\ Department of Mathematics, Freie Universität Berlin, Arnimallee 6, 14195 Berlin, Germany \\ Correspondence should be addressed to Jan-David Hardtke; hardtke@math.fu-berlin.de \\ Received 24 May 2013; Accepted 27 June 2013 \\ Academic Editor: T. S. S. R. K. Rao
}

Copyright (C) 2013 Jan-David Hardtke. This is an open access article distributed under the Creative Commons Attribution License, which permits unrestricted use, distribution, and reproduction in any medium, provided the original work is properly cited.

In 2000, Kadets et al. introduced the notions of acs, luacs, and uacs spaces, which form common generalisations of well-known rotundity and smoothness properties of Banach spaces. In a recent paper, the author introduced some further related notions and investigated the behaviour of these geometric properties under the formation of absolute sums. The present paper is in a sense a continuation of this work. Here we will study the behaviour of the said properties under the formation of Köthe-Bochner spaces, thereby generalising some results of G. Sirotkin on the acs, luacs, and uacs properties of $L^{p}$-Bochner spaces.

\section{Introduction}

We begin with some notation and definitions. Throughout this paper, $X$ denotes a real Banach space, $X^{*}$ its dual, $B_{X}$ its unit ball and $S_{X}$ its unit sphere.

In the next definition, we summarise the most important rotundity properties.

\section{Definition 1. A Banach space $X$ is called}

(i) rotund $(R)$ if for any two elements $x, y \in S_{X}$ the equality $\|x+y\|=2$ implies $x=y$,

(ii) locally uniformly rotund (LUR) if for every $x \in S_{X}$, the implication

$$
\left\|x_{n}+x\right\| \longrightarrow 2 \Longrightarrow\left\|x_{n}-x\right\| \longrightarrow 0
$$

holds for every sequence $\left(x_{n}\right)_{n \in \mathbb{N}}$ in $S_{X}$,

(iii) weakly locally uniformly rotund (WLUR) if for every $x \in S_{X}$ and every sequence $\left(x_{n}\right)_{n \in \mathbb{N}}$ in $S_{X}$ one has

$$
\left\|x_{n}+x\right\| \longrightarrow 2 \Longrightarrow x_{n} \longrightarrow x \text { weakly, }
$$

(iv) uniformly rotund (UR) if for any two sequences $\left(x_{n}\right)_{n \in \mathbb{N}}$ and $\left(y_{n}\right)_{n \in \mathbb{N}}$ in $S_{X}$ the implication

$$
\left\|x_{n}+y_{n}\right\| \longrightarrow 2 \Longrightarrow\left\|x_{n}-y_{n}\right\| \longrightarrow 0
$$

holds,

(v) weakly uniformly rotund (WUR) if for any two sequences $\left(x_{n}\right)_{n \in \mathbb{N}}$ and $\left(y_{n}\right)_{n \in \mathbb{N}}$ the following implication holds:

$$
\left\|x_{n}+y_{n}\right\| \longrightarrow 2 \Longrightarrow x_{n}-y_{n} \longrightarrow 0 \text { weakly. }
$$

Figure 1 shows the obvious implications between these notions. No other implications are valid in general (see the examples in [1]). Note, however, that all these notions coincide in finite-dimensional spaces, by the compactness of $B_{X}$.

The modulus of convexity of the space $X$ is defined by

$$
\delta_{X}(\varepsilon)=\inf \left\{1-\frac{1}{2\|x+y\|}: x, y \in B_{X},\|x-y\| \geq \varepsilon\right\}
$$

for every $\varepsilon$ in the interval $] 0,2]$. Then $X$ is UR if and only if $\delta_{X}(\varepsilon)>0$ for all $0<\varepsilon \leq 2$.

For the local version one defines

$$
\delta_{X}(x, \varepsilon)=\inf \left\{1-\frac{1}{2\|x+y\|}: y \in B_{X},\|x-y\| \geq \varepsilon\right\}
$$

for every $x \in S_{X}$ and each $\left.\left.\varepsilon \in\right] 0,2\right]$. Then $X$ is LUR if and only if $\delta_{X}(x, \varepsilon)>0$ for all $x \in S_{X}$ and all $0<\varepsilon \leq 2$. 


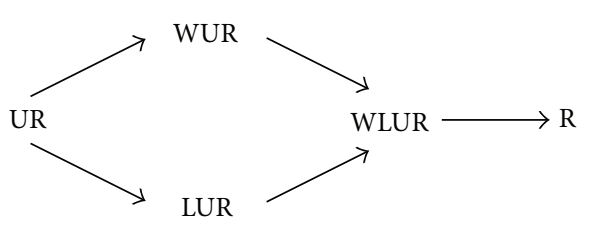

FIGURE 1

Let us also recall some notions of smoothness. The space $X$ is called smooth $(S)$ if its norm is Gâteaux-differentiable at every nonzero point (equivalently at every point of $S_{X}$ ), which is the case if and only if for every $x \in S_{X}$ there is a unique functional $x^{*} \in S_{X^{*}}$ with $x^{*}(x)=1$ (cf. [2, Lemma 8.4 (ii)]). $X$ is called Fréchet-smooth (FS) if the norm is Fréchet-differentiable at every nonzero point. The norm of the space $X$ is said to be uniformly Gâteaux-differentiable (UG) if for each $y \in S_{X}$ the $\operatorname{limit}_{\tau \rightarrow 0}(\|x+\tau y\|-1) / \tau$ exists uniformly in $x \in S_{X}$. Finally, $X$ is called uniformly smooth (US) if $\lim _{\tau \rightarrow 0} \rho_{X}(\tau) / \tau=0$, where $\rho_{X}$ denotes the modulus of smoothness of $X$ defined by $\rho_{X}(\tau)=\sup \{1 / 2(\|x+\tau y\|+$ $\left.\|x-\tau y\|-2): x, y \in S_{X}\right\}$ for every $\tau>0$.

In [3] the following notions were introduced (in connection with the so called Anti-Daugavet property).

Definition 2. A Banach space $X$ is called

(i) alternatively convex or smooth (acs) if for every $x, y \in$ $S_{X}$ with $\|x+y\|=2$ and every $x^{*} \in S_{X^{*}}$ with $x^{*}(x)=1$ one has $x^{*}(y)=1$ as well,

(ii) locally uniformly alternatively convex or smooth (luacs) if for every $x \in S_{X}$, every sequence $\left(x_{n}\right)_{n \in \mathbb{N}}$ in $S_{X}$ and every functional $x^{*} \in S_{X^{*}}$ one has

$$
\left\|x_{n}+x\right\| \longrightarrow 2, \quad x^{*}\left(x_{n}\right) \longrightarrow 1 \Longrightarrow x^{*}(x)=1
$$

(iii) uniformly alternatively convex or smooth (uacs) if for all sequences $\left(x_{n}\right)_{n \in \mathbb{N}},\left(y_{n}\right)_{n \in \mathbb{N}}$ in $S_{X}$ and $\left(x_{n}^{*}\right)_{n \in \mathbb{N}}$ in $S_{X^{*}}$, one has

$\left\|x_{n}+y_{n}\right\| \longrightarrow 2, \quad x_{n}^{*}\left(x_{n}\right) \longrightarrow 1 \Longrightarrow x_{n}^{*}\left(y_{n}\right) \longrightarrow 1$.

The author introduced the following related notions in $[4]$.

Definition 3. A Banach space $X$ is called

(i) strongly locally uniformly alternatively convex or smooth (sluacs) if for every $x \in S_{X}$ and all sequences $\left(x_{n}\right)_{n \in \mathbb{N}}$ in $S_{X}$ and $\left(x_{n}^{*}\right)_{n \in \mathbb{N}}$ in $S_{X^{*}}$, one has

$\left\|x_{n}+x\right\| \longrightarrow 2, \quad x_{n}^{*}\left(x_{n}\right) \longrightarrow 1 \Longrightarrow x_{n}^{*}(x) \longrightarrow 1$,

(ii) weakly uniformly alternatively convex or smooth (wuacs) if for any two sequences $\left(x_{n}\right)_{n \in \mathbb{N}},\left(y_{n}\right)_{n \in \mathbb{N}}$ in $S_{X}$ and every functional $x^{*} \in S_{X^{*}}$, one has

$\left\|x_{n}+y_{n}\right\| \longrightarrow 2, \quad x^{*}\left(x_{n}\right) \longrightarrow 1 \Longrightarrow x^{*}\left(y_{n}\right) \longrightarrow 1$.

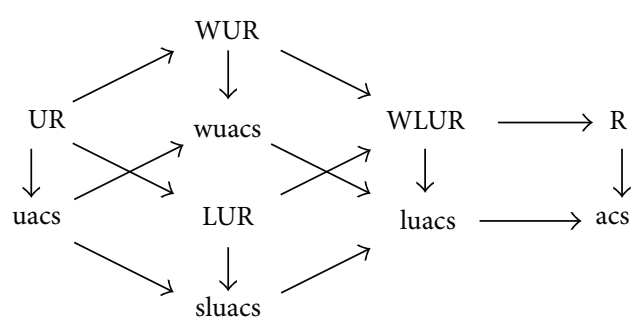

Figure 2

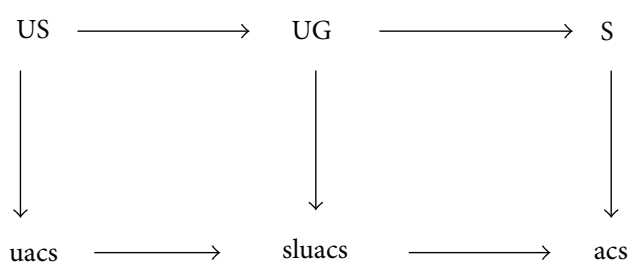

FIGURE 3

The obvious implications between the acs properties and the rotundity properties are indicated in Figure 2. No other implications are generally valid (see the examples in [4]), but note again that the properties acs, luacs, sluacs, wuacs and uacs coincide in finite-dimensional spaces, by compactness.

The connection between some of the acs properties to smoothness properties is illustrated in Figure 3.

Let us mention that if we replace the condition $x_{n}^{*}\left(x_{n}\right) \rightarrow$ 1 by $x_{n}^{*}\left(x_{n}\right)=1$ for every $n \in \mathbb{N}$ in the definitions of the properties uacs and sluacs, respectively we still obtain the same classes of spaces. For uacs spaces this was first proved by Sirotkin in [5] using the fact that uacs spaces are reflexive (see below). For sluacs spaces this characterisation can be proved by means of the Bishop-Phelps-Bollobás theorem (see [4, Proposition 2.1]).

This characterisation enables us to define the following "uacs-modulus" of a given Banach space (cf. [4, Definition $1.4])$.

Definition 4. For a Banach space $X$ one defines

$$
\begin{aligned}
& D_{X}(\varepsilon)=\left\{(x, y) \in S_{X} \times S_{X}: \exists x^{*} \in S_{X^{*}} x^{*}(x)=1,\right. \\
& \left.\qquad x^{*}(y) \leq 1-\varepsilon\right\}, \\
& \left.\left.\delta_{\text {uacs }}^{X}(\varepsilon)=\inf \left\{1-\left\|\frac{x+y}{2}\right\|:(x, y) \in D_{X}(\varepsilon)\right\} \quad \forall \varepsilon \in\right] 0,2\right] .
\end{aligned}
$$

Then $X$ is uacs if and only if $\delta_{\text {uacs }}^{X}(\varepsilon)>0$ for every $\left.\left.\varepsilon \in\right] 0,2\right]$ and one clearly has $\delta_{X}(\varepsilon) \leq \delta_{\text {uacs }}^{X}(\varepsilon)$ for each $\left.\left.\varepsilon \in\right] 0,2\right]$.

The above characterisation shows that the class of uacs spaces coincides with the class of $u$-spaces introduced by Lau in [6] and our modulus $\delta_{\text {uacs }}^{X}$ is the same as the modulus of $u$ convexity from [7]. Also, the notion of $u$-spaces which was introduced in [8] coincides with the notion of acs spaces.

Recall that a Banach space $X$ is said to be uniformly nonsquare if there is some $\delta>0$ such that for all $x, y \in B_{X}$ 
we have $\|x+y\| \leq 2(1-\delta)$ or $\|x-y\| \leq 2(1-\delta)$. It is easily seen that uacs spaces are uniformly nonsquare and hence by a well-known theorem of James (cf. [9, page 261]) they are superreflexive, as was observed in [3, Lemma 4.4]. For a proof of the superreflexivity of uacs spaces that does not rely on James' result on uniformly nonsquare spaces, see [4, Proposition 2.8].

Let us also restate here the following auxiliary result [4, Lemma 2.30] (it is the generalisation of [10, Lemma 2.1] to sequences, with a completely analogous proof).

Lemma 5. Let $\left(x_{n}\right)_{n \in \mathbb{N}}$ and $\left(y_{n}\right)_{n \in \mathbb{N}}$ be sequences in the (real or complex) normed space $X$ such that $\left\|x_{n}+y_{n}\right\|-\left\|x_{n}\right\|-\left\|y_{n}\right\| \rightarrow$ 0.

Then for any two bounded sequences $\left(\alpha_{n}\right)_{n \in \mathbb{N}},\left(\beta_{n}\right)_{n \in \mathbb{N}}$ of nonnegative real numbers one also has $\left\|\alpha_{n} x_{n}+\beta_{n} y_{n}\right\|-\alpha_{n}\left\|x_{n}\right\|-$ $\beta_{n}\left\|y_{n}\right\| \rightarrow 0$.

Finally, we will need two more definitions from [4].

Definition 6. A Banach space $X$ is called

(i) a luacs ${ }^{+}$space if for every $x \in S_{X}$, every sequence $\left(x_{n}\right)_{n \in \mathbb{N}}$ in $S_{X}$ with $\left\|x_{n}+x\right\| \rightarrow 2$, and all $x^{*} \in S_{X^{*}}$ one has

$$
x^{*}\left(x_{n}\right) \longrightarrow 1 \Longleftrightarrow x^{*}(x)=1,
$$

(ii) a sluacs ${ }^{+}$space if for every $x \in S_{X}$, every sequence $\left(x_{n}\right)_{n \in \mathbb{N}}$ in $S_{X}$ with $\left\|x_{n}+x\right\| \rightarrow 2$, and all sequences $\left(x_{n}^{*}\right)_{n \in \mathbb{N}}$ in $S_{X^{*}}$, one has

$$
x_{n}^{*}\left(x_{n}\right) \longrightarrow 1 \Longleftrightarrow x_{n}^{*}(x) \longrightarrow 1 .
$$

Obviously, every WLUR space is luacs ${ }^{+}$, and every LUR space is sluacs ${ }^{+}$.

In the next section we will recall some facts on KötheBochner spaces.

\section{Preliminaries on Köthe-Bochner Spaces}

If not otherwise stated, $(S, \mathscr{A}, \mu)$ will denote a complete, $\sigma$ finite measure space. For $A \in \mathscr{A}$ we denote by $\chi_{A}$ the characteristic function of $A$.

A Köthe function space over $(S, \mathscr{A}, \mu)$ is a Banach space $\left(E,\|\cdot\|_{E}\right)$ of real-valued measurable (i.e., $\mathscr{A}$-Borelmeasurable) functions on $S$ modulo equality $\mu$-almost everywhere (we will henceforth abbreviate this by $\mu$-a.e. or simply a.e. if $\mu$ is tacitly understood) such that

(i) $\chi_{A} \in E$ for every $A \in \mathscr{A}$ with $\mu(A)<\infty$,

(ii) for every $f \in E$ and every set $A \in \mathscr{A}$ with $\mu(A)<$ $\infty f$ is $\mu$-integrable over $A$,

(iii) if $g$ is measurable and $f \in E$ such that $|g(t)| \leq$ $|f(t)| \mu$-a.e., then $g \in E$ and $\|g\|_{E} \leq\|f\|_{E}$.

The standard examples are of course the spaces $L^{p}(\mu)$ for $1 \leq p \leq \infty$.
Every Köthe function space $E$ is a Banach lattice when endowed with the natural order $f \leq g$ if and only if $f(t) \leq$ $g(t) \mu$-a.e.

Recall that a Banach lattice $E$ is said to be order complete ( $\sigma$-order complete) if for every net (sequence) in $E$ which is order bounded, the supremum of said net (sequence) in $E$ exists. A Banach lattice $E$ is called order continuous ( $\sigma$-order continuous) provided that every decreasing net (sequence) in $E$ whose infimum is zero is norm-convergent to zero.

It is easy to see that a Köthe function space $E$ is always $\sigma$ order complete, and thus by [11, Proposition 3.1.5] $E$ is order continuous if and only if $E$ is $\sigma$-order continuous if and only if $E$ is order complete and order continuous. Also, reflexivity of $E$ implies order continuity, for any $\sigma$-order complete Banach lattice which is not $\sigma$-order continuous contains an isomorphic copy of $\ell^{\infty}$ (cf. [11, Proposition 3.1.4]).

Let us also mention the following well-known fact that will be needed later.

Lemma 7. If $E$ is a Köthe function space, $\left(f_{n}\right)_{n \in \mathbb{N}}$ a sequence in $E$, and $f \in E$ such that $\left\|f_{n}-f\right\|_{E} \rightarrow 0$, then there is a subsequence of $\left(f_{n}\right)_{n \in \mathbb{N}}$ which converges pointwise almost everywhere to $f$.

For a Köthe function space $E$ we denote by $E^{\prime}$ the space of all measurable functions $g: S \rightarrow \mathbb{R}$ (modulo equality $\mu$-a.e.) such that

$$
\|g\|_{E^{\prime}}:=\sup \left\{\int_{S}|f g| \mathrm{d} \mu: f \in B_{E}\right\}<\infty .
$$

Then $\left(E^{\prime},\|\cdot\|_{E^{\prime}}\right)$ is again a Köthe function space, the so called Köthe dual of $E$. The operator $T: E^{\prime} \rightarrow E^{*}$ defined by

$$
(T g)(f)=\int_{S} f g \mathrm{~d} \mu \quad \forall f \in E, \forall g \in E^{\prime}
$$

is well-defined, linear and isometric. Moreover, $T$ is surjective if and only if $E$ is order continuous (cf. [11, page 149]), thus for order continuous $E$ we have $E^{*}=E^{\prime}$.

We refer the reader to [12] or [11] for more information on Banach lattices in general and Köthe function spaces in particular.

Now recall that if $X$ is a Banach space, a function $f$ : $S \rightarrow X$ is called simple if there are finitely many disjoint measurable sets $A_{1}, \ldots, A_{n} \in \mathscr{A}$ such that $\mu\left(A_{i}\right)<\infty$ for all $i=1, \ldots, n, f$ is constant on each $A_{i}$ and $f(t)=0$ for every $t \in S \backslash \bigcup_{i=1}^{n} A_{i}$. The function $f$ is said to be Bochner-measurable if there exists a sequence $\left(f_{n}\right)_{n \in \mathbb{N}}$ of simple functions such that $\lim _{n \rightarrow \infty}\left\|f_{n}(t)-f(t)\right\|=0 \mu$ a.e. and weakly measurable if $x^{*} \circ f$ is measurable for every functional $x^{*} \in X^{*}$. According to Pettis' measurability theorem (cf. [11, Theorem 3.2.2]) $f$ is Bochner-measurable if and only if $f$ is weakly measurable and almost everywhere separably valued (i.e., there is a separable subspace $Y \subseteq X$ such that $f(t) \in Y \mu$-a.e.).

For a Köthe function space $E$ and a Banach space $X$ we denote by $E(X)$ the space of all Bochner-measurable functions $f: S \rightarrow X$ (modulo equality a.e.) such that $\|f(\cdot)\| \in E$. Endowed with the norm $\|f\|_{E(X)}=\|\| f(\cdot)\|\|_{E} E(X)$ becomes 
a Banach space, the so called Köthe Bochner space induced by $E$ and $X$. The most prominent examples are again the Lebesgue-Bochner spaces $L^{p}(X)$ for $1 \leq p \leq \infty$.

Next we recall how the dual of $E(X)$ can be described provided that $E$ is order continuous. A function $F: S \rightarrow X^{*}$ is called weak ${ }^{*}$-measurable if $F(\cdot)(x)$ is measurable for every $x \in X$. We define an equivalence relation on the set of all weak ${ }^{*}$-measurable functions by setting $F \sim G$ if and only if for every $x \in X F(t)(x)=G(t)(x)$ a.e., and we write $E^{\prime}\left(X^{*}, w^{*}\right)$ for the space of all (equivalence classes of) weak ${ }^{*}$ measurable functions $F$ such that there is some $g \in E^{\prime}$ with $\|F(t)\| \leq g(t)$ a.e.

A norm on $E^{\prime}\left(X^{*}, w^{*}\right)$ can be defined by

$$
\|[F]\|_{E^{\prime}\left(X^{*}, w^{*}\right)}:=\inf \left\{\|g\|_{E^{\prime}}: g \in E^{\prime}, F(t) \leq g(t) \text { a.e. }\right\} .
$$

Then the following deep theorem holds.

Theorem 8 (cf. [13]). Let $E$ be an order-continuous Köthe function space over the complete, $\sigma$-finite measure space $(S, A, \mu)$, and let $X$ be a Banach space. Then the map $V$ : $E^{\prime}\left(X^{*}, w^{*}\right) \rightarrow E(X)^{*}$ defined by

$$
\begin{aligned}
& V([F])(f) \\
& :=\int_{S} F(t)(f(t)) \mathrm{d} \mu(t), \quad \forall f \in E(X), \quad \forall[F] \in E^{\prime}\left(X^{*}, w^{*}\right)
\end{aligned}
$$

is an isometric isomorphism, and, moreover, every equivalence class $L$ in $E^{\prime}\left(X^{*}, w^{*}\right)$ has a representative $F$ such that $\|F(\cdot)\| \epsilon$ $E^{\prime}$ and $\|L\|_{E^{\prime}\left(X^{*}, w^{*}\right)}=\|\| F(\cdot)\|\|_{E^{\prime}}$.

There are a number of papers on various rotundity and smoothness properties in Köthe-Bochner spaces in general and Lebesgues-Bochner spaces in particular, see for example [14-17] and references therein.

Sirotkin proved in [5] that for $1<p<\infty$ the LebesgueBochner space $L^{p}(X)$ is acs, respectively luacs, and uacs, whenever $X$ has the respective property. In the next section we will study the more general case of Köthe-Bochner spaces.

\section{Results and Proofs}

We begin with the acs spaces, for which we have the following result.

Proposition 9. If $E$ is an order-continuous acs Köthe function space and $X$ is an acs Banach space, then $E(X)$ is acs as well.

Proof. The proof is similar to that of [4, Proposition 3.3]. First we fix two elements $f, g \in S_{E(X)}$ such that $\|f+g\|_{E(X)}=2$ and a functional $l \in S_{E(X)^{*}}$ with $l(f)=1$.

Since $E$ is order continuous, by Theorem $8, l$ can be represented via an element $[F] \in E^{\prime}\left(X^{*}, w^{*}\right)$ such that
$\|F(\cdot)\| \in E^{\prime}$ and \|\|$F(\cdot)\|\|_{E^{\prime}}=\|[F]\|_{E^{\prime}\left(X^{*}, w^{*}\right)}=\|l\|=1$. It follows that

$$
\begin{aligned}
1 & =l(f)=\int_{S} F(t)(f(t)) \mathrm{d} \mu(t) \\
& \leq \int_{S}\|F(t)\|\|f(t)\| \mathrm{d} \mu(t) \leq\|\| F(\cdot)\|\|_{E^{\prime}}\|\| f(\cdot)\|\|_{E} \\
& =\|l\|\|f\|_{E(X)}=1
\end{aligned}
$$

and hence

$$
\begin{gathered}
\int_{S}\|F(t)\|\|f(t)\| \mathrm{d} \mu(t)=1, \\
F(t)(f(t))=\|F(t)\|\|f(t)\| \quad \text { a.e. }
\end{gathered}
$$

We also have

$$
\begin{aligned}
2 & =\|f+g\|_{E(X)}=\|\| f(\cdot)+g(\cdot)\|\|_{E} \\
& \leq\|\| f(\cdot)\|+\| g(\cdot)\|\|_{E} \leq\|f\|_{E(X)}+\|g\|_{E(X)}=2
\end{aligned}
$$

and thus

$$
\|\| f(\cdot)\|+\| g(\cdot)\|\|_{E}=2 .
$$

Since $E$ is acs it follows from (19) and (22) that

$$
\int_{S}\|F(t)\|\|g(t)\| \mathrm{d} \mu(t)=1 .
$$

In a similar way as we have obtained (22) we can also show

$$
\|\| f(\cdot)+g(\cdot)\|+\| f(\cdot)\|+\| g(\cdot)\|\|_{E}=4 .
$$

Because $E$ is acs this together with (19), (22) and (23) implies

$$
\int_{S}\|F(t)\|\|f(t)+g(t)\| \mathrm{d} \mu(t)=2 .
$$

From (19), (23) and (25) we get

$$
\|F(t)\|(\|f(t)\|+\|g(t)\|-\|f(t)+g(t)\|)=0 \quad \text { a.e. }
$$

Now we will show that

$$
F(t)(g(t))=\|F(t)\|\|g(t)\| \quad \text { a.e. }
$$

To this end, let us denote by $N_{1}$ and $N_{2}$ the null sets on which the equality from (20) and (26), respectively does not hold. Let $N=N_{1} \cup N_{2}$.

Put $B=\{t \in S \backslash N: F(t) \neq 0$ and $g(t) \neq 0\}$ and $C=\{t \in$ $B: f(t)=0\}$. We claim that $C$ is a null set.

To see this, define $h: S \rightarrow \mathbb{R}$ by $h(t)=\|F(t)\|$ for $t \in S \backslash C$ and $h(t)=0$ for $t \in C$. Then $h$ is measurable and since $h(t) \leq$ $\|F(t)\|$ for all $t \in S$ we have $h \in E^{\prime}$ with $\|h\|_{E^{\prime}} \leq 1$. We also have $h(t)\|f(t)\|=\|F(t)\|\|f(t)\|$ for every $t \in S$ and hence by (19)

$$
\int_{S} h(t)\|f(t)\| \mathrm{d} \mu(t)=1
$$


which also implies $\|h\|_{E^{\prime}}=1$. Together with (22) we now get

$$
\int_{S} h(t)\|g(t)\| \mathrm{d} \mu(t)=1,
$$

since $E$ is acs. Taking into account (23) we arrive at

$$
\int_{S}(\|F(t)\|-h(t))\|g(t)\| \mathrm{d} \mu(t)=0 .
$$

Hence $(\|F(t)\|-h(t))\|g(t)\|=0$ a.e. and thus $C$ must be a null set.

Now if $t \in(S \backslash C) \cap B$ then $F(t) \neq 0, f(t) \neq 0$ and $g(t) \neq 0$ and $\|F(t)\|\|f(t)\|=F(t)(f(t))$ as well as

$$
\|f(t)+g(t)\|=\|f(t)\|+\|g(t)\| .
$$

By [10, Lemma 2.1] this implies

$$
\left\|\frac{f(t)}{\|f(t)\|}+\frac{g(t)}{\|g(t)\|}\right\|=2 .
$$

Since $X$ is acs it follows that $\|F(t)\|\|g(t)\|=F(t)(g(t))$.

So $M:=N \cup C$ is a null set with $\|F(t)\|\|g(t)\|=F(t)(g(t))$ for every $t \in S \backslash M$ and (27) is proved.

Now combining (23) and (27) we obtain

$$
l(g)=\int_{S} F(t)(g(t)) \mathrm{d} \mu(t)=1,
$$

which finishes the proof.

Before we turn to the case of luacs spaces, let us recall Egorov's theorem (cf. [18, Theorem A, page 88]), which states that for any finite measure space $(S, \mathscr{A}, \mu)$ and every sequence $\left(f_{n}\right)_{n \in \mathbb{N}}$ of measurable functions on $S$ which converges to zero pointwise $\mu$-a.e. and each $\varepsilon>0$ there is a set $A \in \mathscr{A}$ with $\mu(S \backslash A) \leq \varepsilon$ such that $\left(f_{n}\right)_{n \in \mathbb{N}}$ is uniformly convergent to zero on $A$.

Now we are ready to prove the following theorem.

Theorem 10. Let $E$ be an order-continuous Köthe function space over the complete $\sigma$-finite measure space $(S, \mathscr{A}, \mu)$ and $X$ an luacs Banach space. If

(a) $E$ is WLUR or

(b) $E$ is luacs ${ }^{+}$and $E^{\prime}$ is also order continuous,

then $E(X)$ is also luacs.

Proof. Suppose that we are given a sequence $\left(f_{n}\right)_{n \in \mathbb{N}}$ in $S_{E(X)}$ and an element $f \in S_{E(X)}$ such that $\left\|f_{n}+f\right\|_{E(X)} \rightarrow 2$ as well as a functional $l \in S_{E(X)^{*}}$ such that $l\left(f_{n}\right) \rightarrow 1$. As before, we can represent $l$ by an element $[F] \in E^{\prime}\left(X^{*}, w^{*}\right)$. We then have

$$
l\left(f_{n}\right)=\int_{S} F(t)\left(f_{n}(t)\right) \mathrm{d} \mu(t) \leq \int_{S}\|F(t)\|\left\|f_{n}(t)\right\| \mathrm{d} \mu(t) \leq 1
$$

and hence

$$
\lim _{n \rightarrow \infty} \int_{S}\|F(t)\|\left\|f_{n}(t)\right\| \mathrm{d} \mu(t)=1 .
$$

By passing to a subsequence we may also assume that

$$
\lim _{n \rightarrow \infty}\left(\|F(t)\|\left\|f_{n}(t)\right\|-F(t)\left(f_{n}(t)\right)\right)=0 \quad \text { a.e. }
$$

We further have

$$
\left\|f_{n}+f\right\|_{E(X)}=\|\| f_{n}(\cdot)+f(\cdot)\|\|_{E} \leq\|\| f_{n}(\cdot)\|+\| f(\cdot)\|\|_{E} \leq 2
$$

and thus

$$
\lim _{n \rightarrow \infty}\|\| f_{n}(\cdot)\|+\| f(\cdot)\|\|_{E}=2 .
$$

An analogous argument also shows

$$
\lim _{n \rightarrow \infty}\|\| f_{n}(\cdot)+f(\cdot)\|+\| f_{n}(\cdot)\|+\| f(\cdot)\|\|_{E}=4 .
$$

Moreover, the inequality

$$
\begin{aligned}
\| f_{n}+ & f \|_{E(X)}+1 \\
& \geq\|\| f_{n}(\cdot)+f(\cdot)\|+\| f_{n}(\cdot)\|\|_{E} \\
& \geq\|\| f_{n}(\cdot)+f(\cdot)\|+\| f_{n}(\cdot)\|+\| f(\cdot)\|\|_{E}-1
\end{aligned}
$$

holds for every $n \in \mathbb{N}$. It follows that

$$
\lim _{n \rightarrow \infty}\|\| f_{n}(\cdot)+f(\cdot)\|+\| f_{n}(\cdot)\|\|_{E}=3 .
$$

Analogously one can see that

$$
\lim _{n \rightarrow \infty}\|\| f_{n}(\cdot)+f(\cdot)\|+\| f(\cdot)\|\|_{E}=3 .
$$

Finally, we have

$$
\begin{aligned}
\|\| f_{n}(\cdot) & +f(\cdot)\|+\| f_{n}(\cdot)\|\|_{E}+3 \\
& \geq\|\| f_{n}(\cdot)+f(\cdot)\|+\| f_{n}(\cdot)\|+3\| f(\cdot)\|\|_{E} \\
& \geq 2\|\| f_{n}(\cdot)+f(\cdot)\|+\| f(\cdot)\|\|_{E} \cdot
\end{aligned}
$$

Consequently

$$
\lim _{n \rightarrow \infty}\|\| f_{n}(\cdot)+f(\cdot)\|+\| f_{n}(\cdot)\|+3\| f(\cdot)\|\|_{E}=6 .
$$

Since $E$ is in particular luacs we get from (35) and (38) that

$$
\int_{S}\|F(t)\|\|f(t)\| \mathrm{d} \mu(t)=1 .
$$

Because $E$ is in any case luacs ${ }^{+}$, it follows from (41), (44), and (45) that

$$
\lim _{n \rightarrow \infty} \int_{S}\|F(t)\|\left(\left\|f_{n}(t)\right\|+\left\|f_{n}(t)+f(t)\right\|\right) \mathrm{d} \mu(t)=3
$$

and thus

$$
\begin{aligned}
\lim _{n \rightarrow \infty} \int_{S}\|F(t)\|\left(\left\|f_{n}(t)\right\|+\|f(t)\|\right. \\
\left.-\left\|f_{n}(t)+f(t)\right\|\right) \mathrm{d} \mu(t)=0 .
\end{aligned}
$$


So by passing to a further subsequence we may assume

$$
\lim _{n \rightarrow \infty}\|F(t)\|\left(\left\|f_{n}(t)\right\|+\|f(t)\|-\left\|f_{n}(t)+f(t)\right\|\right)=0 \quad \text { a.e. }
$$

Next we will show that

$$
F(t)(f(t))=\|F(t)\|\|f(t)\| \quad \text { a.e. }
$$

Since $(S, \mathscr{A}, \mu)$ is $\sigma$-finite there is an increasing sequence $\left(A_{m}\right)_{m \in \mathbb{N}}$ in $\mathscr{A}$ such that $\mu\left(A_{m}\right)<\infty$ for every $m \in \mathbb{N}$ and $\bigcup_{m=1}^{\infty} A_{m}=S$.

Denote by $N_{1}$ and $N_{2}$ the null sets on which the convergence statement from (36) and (48), respectively does not hold, and let $N=N_{1} \cup N_{2}$. Put $B=\{t \in S \backslash N$ : $F(t) \neq 0$ and $f(t) \neq 0\}$ and $C=\left\{t \in B: f_{n}(t) \rightarrow 0\right\}$. We will see that $C$ is a null set.

First we define for every $m \in \mathbb{N}$ a function $a_{m}: S \rightarrow \mathbb{R}$ by setting $a_{m}(t)=\|F(t)\|$ for $t \in S \backslash\left(C \cap A_{m}\right)$ and $a_{m}(t)=0$ for $t \in C \cap A_{m}$. Note that each $a_{m}$ is measurable and since $\left|a_{m}(t)\right| \leq\|F(t)\|$ for every $t \in S$ we have $a_{m} \in B_{E^{\prime}}$.

We have $\lim _{k \rightarrow \infty}\|F(t)\|\left\|f_{k}(t)\right\| \chi_{C \cap A_{m}}(t)=0$ for every $t \in S$ and every $m \in \mathbb{N}$, so by Egorov's theorem we can find for every $m \in \mathbb{N}$ an increasing sequence $\left(B_{n, m}\right)_{n \in \mathbb{N}}$ in $\left.\mathscr{A}\right|_{A_{m}}$ with $\mu\left(A_{m} \backslash B_{n, m}\right) \leq 1 / n$ such that $\left(\|F(\cdot)\|\left\|f_{k}(\cdot)\right\| \chi_{C \cap A_{m}}\right)_{k \in \mathbb{N}}$ converges uniformly to zero on each $B_{n, m}$.

It follows that $M_{m}:=\bigcap_{n=1}^{\infty} A_{m} \backslash B_{n, m}$ is a null set for every $m \in \mathbb{N}$.

Let us now first suppose that (b) holds, so $E^{\prime}$ is order continuous. We have

$$
\lim _{n \rightarrow \infty}\|F(t)\| \chi_{C \cap\left(A_{m} \backslash B_{n, m}\right)}(t)=0 \quad \forall t \in S \backslash M_{m}
$$

and moreover this sequence is decreasing, so the order continuity of $E^{\prime}$ implies

$$
\lim _{n \rightarrow \infty}\|\| F(\cdot)\left\|\chi_{C \cap\left(A_{m} \backslash B_{n, m}\right)}\right\|_{E^{\prime}}=0
$$

So if $m \in \mathbb{N}$ and $\varepsilon>0$ are given, we can find an index $n \in \mathbb{N}$ such that \|\|$F(\cdot)\left\|\chi_{C \cap\left(A_{m} \backslash B_{n, m}\right)}\right\|_{E^{\prime}} \leq \varepsilon$, and then, by uniform convergence, an index $k_{0} \in \mathbb{N}$ such that $\|F(t)\|\left\|f_{k}(t)\right\| \chi_{C \cap B_{n, m}}(t) \leq \varepsilon \mu\left(A_{m}\right)^{-1}$ for every $t \in S$ and every $k \geq k_{0}$.

Then we have

$$
\begin{aligned}
\int_{C \cap A_{m}} & \|F(t)\|\left\|f_{k}(t)\right\| \mathrm{d} \mu(t) \\
= & \int_{C \cap B_{n, m}}\|F(t)\|\left\|f_{k}(t)\right\| \mathrm{d} \mu(t) \\
& +\int_{C \cap\left(A_{m} \backslash B_{n, m}\right)}\|F(t)\|\left\|f_{k}(t)\right\| \mathrm{d} \mu(t) \\
\leq & \int_{C \cap B_{n, m}} \frac{\varepsilon}{\mu\left(A_{m}\right)} \mathrm{d} \mu(t)+\|\| F(\cdot)\left\|\chi_{C \cap\left(A_{m} \backslash B_{n, m}\right)}\right\|_{E^{\prime}} \\
\leq & 2 \varepsilon
\end{aligned}
$$

for each $k \geq k_{0}$.
In conclusion we have

$$
\lim _{k \rightarrow \infty} \int_{C \cap A_{m}}\|F(t)\|\left\|f_{k}(t)\right\| \mathrm{d} \mu(t)=0 \quad \forall m \in \mathbb{N} .
$$

Now if (a) holds, that is, if $E$ is WLUR, then by (38) the sequence $\left(\left\|f_{k}(\cdot)\right\|\right)_{k \in \mathbb{N}}$ must be weakly convergent to $\|f(\cdot)\|$ in $E$, and hence

$$
\begin{array}{r}
\lim _{k \rightarrow \infty} \int_{C \cap\left(A_{m} \backslash B_{n, m}\right)}\|F(t)\|\left\|f_{k}(t)\right\| \mathrm{d} \mu(t) \\
=\int_{C \cap\left(A_{m} \backslash B_{n, m}\right)}\|F(t)\|\|f(t)\| \mathrm{d} \mu(t)
\end{array}
$$

for all $n, m \in \mathbb{N}$. Since $\left(\|f(\cdot)\| \chi_{C \cap\left(A_{m} \backslash B_{n, m}\right)}\right)_{n \in \mathbb{N}}$ decreases to zero a.e., the order continuity of $E$ gives us $\lim _{n \rightarrow \infty}\|\| f(\cdot)\left\|\chi_{C \cap\left(A_{m} \backslash B_{n, m}\right)}\right\|_{E}=0$ for every $m \in \mathbb{N}$.

A similiar argument as before now easily yields that $(+)$ also holds in case (a). But $(+)$ is nothing else than

$$
\lim _{n \rightarrow \infty} \int_{S}\left(\|F(t)\|-a_{m}(t)\right)\left\|f_{n}(t)\right\| \mathrm{d} \mu(t)=0 \quad \forall m \in \mathbb{N} .
$$

Combining this with (35) leaves us with

$$
\lim _{n \rightarrow \infty} \int_{S} a_{m}(t)\left\|f_{n}(t)\right\| \mathrm{d} \mu(t)=1 \quad \forall m \in \mathbb{N} .
$$

Since $E$ is luacs and because of (38), it follows that

$$
\int_{S} a_{m}(t)\|f(t)\| \mathrm{d} \mu(t)=1 \quad \forall m \in \mathbb{N} .
$$

Taking into account (45) we get

$$
\int_{S}\left(\|F(t)\|-a_{m}(t)\right)\|f(t)\| \mathrm{d} \mu(t)=0 \quad \forall m \in \mathbb{N},
$$

and hence for every $m \in \mathbb{N}$ we have $\left(\|F(t)\|-a_{m}(t)\right)\|f(t)\|=0$ a.e. Consequently, $C \cap A_{m}$ is a null set for every $m$, and thus $C=\bigcup_{m=1}^{\infty} C \cap A_{m}$ is also a null set.

Now suppose that $t \in(S \backslash C) \cap B$. Then we have $F(t) \neq 0, f(t) \neq 0$ and $\left\|f_{n}(t)\right\| \nrightarrow 0$, as well as $\|F(t)\|\left\|f_{n}(t)\right\|-$ $F(t)\left(f_{n}(t)\right) \rightarrow 0$ and

$$
\lim _{n \rightarrow \infty}\left(\left\|f_{n}(t)\right\|+\|f(t)\|-\left\|f_{n}(t)+f(t)\right\|\right)=0 .
$$

By passing to a subsequence we may assume that $\left(\left\|f_{n}(t)\right\|\right)_{n \in \mathbb{N}}$ is bounded away from zero. Then it follows from Lemma 5 that

$$
\lim _{n \rightarrow \infty}\left\|\frac{f_{n}(t)}{\left\|f_{n}(t)\right\|}+\frac{f(t)}{\|f(t)\|}\right\|=2 .
$$

Also, we have

$$
\lim _{n \rightarrow \infty} \frac{F(t)}{\|F(t)\|}\left(\frac{f_{n}(t)}{\left\|f_{n}(t)\right\|}\right)=1 .
$$

Since $X$ is luacs we can conclude that $F(t)(f(t))=$ $\|F(t)\|\|f(t)\|$. 
So $M:=N \cup C$ is a null set with $F(t)(f(t))=\|F(t)\|\|f(t)\|$ for every $t \in S \backslash M$ and (49) is proved.

From (45) and (49) it follows that

$$
l(f)=\int_{S} F(t)(f(t)) \mathrm{d} \mu(t)=1
$$

and we are done.

Recall that a subset $A \subseteq L^{1}(\mu)$ is said to be equi-integrable if for every $\varepsilon>0$ there is some $\delta>0$ such that

$$
B \in \mathscr{A} \text { with } \mu(B) \leq \delta \Longrightarrow\left|\int_{B} f \mathrm{~d} \mu\right| \leq \varepsilon \quad \forall f \in A .
$$

It is well known that for a finite measure $\mu$ a bounded subset $A \subseteq L^{1}(\mu)$ is relatively weakly compact in $L^{1}(\mu)$ if and only if $A$ is equi-integrable (see, e.g., [19, Theorem 13.6]). One ingredient for the usual proof of this fact is the following lemma (see [19, Proposition 13.4]), which we will also need in the sequel.

Lemma 11. For a finite measure space $(S, \mathscr{A}, \mu)$, a sequence $\left(f_{n}\right)_{n \in \mathbb{N}}$ in $L^{1}(\mu)$ is equi-integrable whenever the sequence $\left(\int_{B} f_{n} \mathrm{~d} \mu\right)_{n \in \mathbb{N}}$ is convergent for each $B \in \mathscr{A}$.

We will also need Vitali's lemma, which reads as follows (see, e.g., [11, Lemma 3.1.13] for an even more general version).

Lemma 12. Let $(S, \mathscr{A}, \mu)$ be a finite measure space, and let $\left(f_{n}\right)_{n \in \mathbb{N}}$ be a sequence in $L^{1}(\mu)$ such that $\left\{\left|f_{n}\right|: n \in \mathbb{N}\right\}$ is equi-integrable. Let $f$ be a measurable function on $S$ such that $f_{n}(t) \rightarrow f(t) \mu$-a.e. Then $f \in L^{1}(\mu)$ and $\left\|f_{n}-f\right\|_{1} \rightarrow 0$.

Finally, let us recall that a Banach space $X$ is said to have the Kadets-Klee property (also known as property $(H)$ ) if for every sequence $\left(x_{n}\right)_{n \in \mathbb{N}}$ in $X$ and each $x \in X$ the implication

$$
x_{n} \stackrel{\sigma}{\longrightarrow} x, \quad\left\|x_{n}\right\| \longrightarrow\|x\| \Longrightarrow\left\|x_{n}-x\right\| \longrightarrow 0
$$

holds. For example, every LUR space and every dual of a reflexive, FS space has the Kadets-Klee property.

It is known that every Banach lattice with the Kadets-Klee property is order continuous, (cf. [12, page 28]). With this in mind we can prove the following result concerning luacs ${ }^{+}$ spaces.

Theorem 13. If the measure $\mu$ is finite and $E$ is $L U R$, then $E(X)$ is a luacs ${ }^{+}$space whenever $X$ is luacs ${ }^{+}$. If in addition $E^{\prime}$ is order continuous then the assertion also holds if $\mu$ is merely $\sigma$-finite.

Proof. By the previous theorem, $E(X)$ is luacs, so we only have to show the implication " $\models$ " in Definition 6 (i). To this end, let $\left(f_{n}\right)_{n \in \mathbb{N}}$ be a sequence in $S_{E(X)}$ and $f \in S_{E(X)}$ such that $\left\|f_{n}+f\right\|_{E(X)} \rightarrow 2$, and let $l \in S_{E(X)^{*}}$ such that $l(f)=1$. It will be enough to show that a subsequence of $\left(l\left(f_{n}\right)\right)_{n \in \mathbb{N}}$ converges to one.
Since $E$ is order continuous, we can as before represent $l$ by some $[F] \in E^{\prime}\left(X^{*}, w^{*}\right)$ and conclude

$$
\begin{gathered}
\int_{S}\|F(t)\|\|f(t)\| \mathrm{d} \mu(t)=1, \\
\|F(t)\|\|f(t)\|=F(t)(f(t)) \quad \text { a.e. }
\end{gathered}
$$

Also, just as we have done in the previous proof, we find that

$$
\begin{gathered}
\lim _{n \rightarrow \infty}\|\| f_{n}(\cdot)\|+\| f(\cdot)\|\|_{E}=2, \\
\lim _{n \rightarrow \infty}\|\| f_{n}(\cdot)+f(\cdot)\|+\| f_{n}(\cdot)\|+\| f(\cdot)\|\|_{E}=4, \\
\lim _{n \rightarrow \infty}\|\| f_{n}(\cdot)+f(\cdot)\|+\| f_{n}(\cdot)\|\|_{E}=3, \\
\lim _{n \rightarrow \infty}\|\| f_{n}(\cdot)+f(\cdot)\|+\| f(\cdot)\|\|_{E}=3, \\
\lim _{n \rightarrow \infty}\|\| f_{n}(\cdot)+f(\cdot)\|+\| f_{n}(\cdot)\|+3\| f(\cdot)\|\|_{E}=6 .
\end{gathered}
$$

Since $E$ is LUR, it follows that

$$
\begin{gathered}
\lim _{n \rightarrow \infty}\|\| f_{n}(\cdot)\|-\| f(\cdot)\|\|_{E}=0, \\
\lim _{n \rightarrow \infty}\|\| f_{n}(\cdot)+f(\cdot)\|+\| f_{n}(\cdot)\|-3\| f(\cdot)\|\|_{E}=0 .
\end{gathered}
$$

Hence, by passing to a subsequence, we may assume that (cf. Lemma 7)

$$
\begin{gathered}
\lim _{n \rightarrow \infty}\left\|f_{n}(t)\right\|=\|f(t)\| \quad \text { a.e., } \\
\lim _{n \rightarrow \infty}\left\|f_{n}(t)+f(t)\right\|=2\|f(t)\| \quad \text { a.e. }
\end{gathered}
$$

By (67) and (64) we also have

$$
\lim _{n \rightarrow \infty} \int_{S}\|F(t)\|\left\|f_{n}(t)\right\| \mathrm{d} \mu(t)=1 .
$$

Since $X$ is luacs ${ }^{+}$, it follows from (65), (69) that

$$
\lim _{n \rightarrow \infty}\left(\|F(t)\|\left\|f_{n}(t)\right\|-F(t)\left(f_{n}(t)\right)\right)=0 \quad \text { a.e. }
$$

From (67) we also get

$$
\begin{aligned}
\lim _{n \rightarrow \infty} & \int_{A}\|F(t)\|\left\|f_{n}(t)\right\| \mathrm{d} \mu(t) \\
& =\int_{A}\|F(t)\|\|f(t)\| \mathrm{d} \mu(t) \quad \forall A \in \mathscr{A} .
\end{aligned}
$$

Thus by Lemma 11 the sequence $\left(\|F(\cdot)\|\left\|f_{n}(\cdot)\right\| \chi_{B}\right)_{n \in \mathbb{N}}$ and hence also the sequence $\left(\|\left(F(\cdot)\|\| f_{n}(\cdot) \|-F(\cdot)\left(f_{n}(\cdot)\right)\right) \chi_{B}\right)_{n \in \mathbb{N}}$ are equi-integrable with respect to $\left(B,\left.\mathscr{A}\right|_{B}, \mu_{\left.\mathscr{A}\right|_{B}}\right)$ for every $B \in \mathscr{A}$ with $\mu(B)<\infty$. This combined with Vitali's lemma and (71) implies

$$
\begin{array}{r}
\operatorname{Lim}_{n \rightarrow \infty} \int_{B}\left(\|F(t)\|\left\|f_{n}(t)\right\|-F(t)\left(f_{n}(t)\right)\right) \mathrm{d} \mu(t)=0 \\
\forall B \in \mathscr{A} \text { with } \mu(B)<\infty .
\end{array}
$$


So if $\mu(S)<\infty$, we immediately get

$$
l\left(f_{n}\right)=\int_{S} F(t)\left(f_{n}(t)\right) \mathrm{d} \mu(t) \longrightarrow 1,
$$

because of (70).

If $\mu$ is merely $\sigma$-finite but $E^{\prime}$ is order continuous, we can fix an increasing sequence $\left(A_{m}\right)_{m \in \mathbb{N}}$ in $\mathscr{A}$ such that $\bigcup_{m=1}^{\infty} A_{m}=S$ and $\mu\left(A_{m}\right)<\infty$ for every $m \in \mathbb{N}$. Then the sequence $\left(\|F(\cdot)\| \chi_{S \backslash A_{m}}\right)_{m \in \mathbb{N}}$ decreases pointwise to zero, and, by the order continuity of $E^{\prime}$, we can conclude that \|\|$F(\cdot)\left\|\chi_{S \backslash A_{m}}\right\|_{E^{\prime}} \rightarrow 0$.

Thus given any $\varepsilon>0$, we find an $m_{0} \in \mathbb{N}$ such that \|\|$F(\cdot)\left\|\chi_{S \backslash A_{m_{0}}}\right\|_{E^{\prime}} \leq \varepsilon / 3$. Since $\mu\left(A_{m_{0}}\right)<\infty$, there exists $N \in \mathbb{N}$ such that

$$
\int_{A_{m_{0}}}\left(\|F(t)\|\left\|f_{n}(t)\right\|-F(t)\left(f_{n}(t)\right)\right) \mathrm{d} \mu(t) \leq \frac{\varepsilon}{3} \quad \forall n \geq N .
$$

It follows that for every $n \geq N$

$$
\begin{aligned}
\int_{S}\left(\|F(t)\|\left\|f_{n}(t)\right\|-F(t)\left(f_{n}(t)\right)\right) \mathrm{d} \mu(t) \\
\leq \frac{\varepsilon}{3}+2 \int_{S \backslash A_{m_{0}}}\|F(t)\|\left\|f_{n}(t)\right\| \mathrm{d} \mu(t) \\
\quad \leq \frac{\varepsilon}{3}+2\|\| F(\cdot)\left\|\chi_{S \backslash A_{m_{0}}}\right\|_{E^{\prime}} \leq \varepsilon
\end{aligned}
$$

So we have

$$
\lim _{n \rightarrow \infty} \int_{S}\left(\|F(t)\|\left\|f_{n}(t)\right\|-F(t)\left(f_{n}(t)\right)\right) \mathrm{d} \mu(t)=0,
$$

and because of (70) it follows as before that

$$
l\left(f_{n}\right)=\int_{S} F(t)\left(f_{n}(t)\right) \mathrm{d} \mu(t) \longrightarrow 1,
$$

finishing the proof.

Now we turn to the sluacs spaces. An easy normalisation argument shows that a Banach space $X$ is sluacs if and only if for every $x \in S_{X}$, every sequence $\left(x_{n}^{*}\right)_{n \in \mathbb{N}}$ in $S_{X^{*}}$, and all sequences $\left(x_{n}\right)_{n \in \mathbb{N}}$ in $X$ with $\left\|x_{n}+x\right\| \rightarrow 2,\left\|x_{n}\right\| \rightarrow 1$ and $x_{n}^{*}\left(x_{n}\right) \rightarrow 1$, we have $x_{n}^{*}(x) \rightarrow 1$. In view of this characterisation, $X$ is sluacs if and only if for every $x \in S_{X}$ and every $0<\varepsilon \leq 2$ the number

$$
\begin{array}{r}
\beta_{X}(x, \varepsilon):=\inf \left\{\operatorname { m a x } \left\{1-\left\|\frac{x+y}{2}\right\|,|\|y\|-1|,\right.\right. \\
\left.\left.\left|x^{*}(y)-1\right|\right\}:\left(y, x^{*}\right) \in V_{x, \varepsilon}\right\}
\end{array}
$$

is strictly positive, where

$$
V_{x, \varepsilon}:=\left\{\left(y, x^{*}\right) \in X \times S_{X^{*}}: x^{*}(y-x) \geq \varepsilon\right\} .
$$

Next we will prove an easy lemma on the continuity of $\beta_{X}$.
Lemma 14. For all $0<\varepsilon, \widetilde{\varepsilon}, \leq 2$ and all $x, \tilde{x} \in S_{X}$, one has

$$
\left|\beta_{X}(x, \varepsilon)-\beta_{X}(\widetilde{x}, \widetilde{\varepsilon})\right| \leq\|x-\widetilde{x}\|+|\varepsilon-\widetilde{\varepsilon}|
$$

that is $\beta_{X}$ is 1-Lipschitz continuous with respect to the norm of $X \oplus_{1} \mathbb{R}$.

Proof. First we fix $0<\varepsilon \leq 2$ and $x, \tilde{x} \in S_{X}$. Put $\delta=\|x-\tilde{x}\|$ and take $y \in X, x^{*} \in S_{X^{*}}$ such that $x^{*}(y-x) \geq \varepsilon$. It follows that $x^{*}(y-\tilde{x}) \geq \varepsilon-\delta$.

Now let $0<\tau<1$ be arbitrary. We can find $z \in S_{X}$ with $x^{*}(z) \geq 1-\tau$. Define $\tilde{y}=y+\delta(1-\tau)^{-1} z$. Then

$$
x^{*}(\tilde{y}-\tilde{x})=\frac{\delta}{1-\tau} x^{*}(z)+x^{*}(y-\tilde{x}) \geq \delta+x^{*}(y-\tilde{x})=\varepsilon
$$

and hence

$$
\max \left\{1-\left\|\frac{\tilde{x}+\tilde{y}}{2}\right\|,|\|\tilde{y}\|-1|,\left|x^{*}(\tilde{y})-1\right|\right\} \geq \beta_{X}(\tilde{x}, \varepsilon) .
$$

But we have $|\|\tilde{y}\|-\|y\|| \leq\|y-\tilde{y}\|=\delta(1-\tau)^{-1}$ and $\mid x^{*}(\tilde{y})-$ $x^{*}(y) \mid \leq\|y-\tilde{y}\|=\delta(1-\tau)^{-1}$ as well as

$$
\begin{aligned}
& \mid\left\|\frac{x+y}{2}\right\|-\left\|\frac{\tilde{x}+\tilde{y}}{2}\right\| \\
& \quad \leq \frac{1}{2}(\|x-\tilde{x}\|+\|y-\tilde{y}\|)=\frac{1}{2}\left(\delta+\frac{\delta}{1-\tau}\right) \leq \frac{\delta}{1-\tau} .
\end{aligned}
$$

Thus we get

$$
\begin{aligned}
\max & \left\{1-\left\|\frac{x+y}{2}\right\|,|\|y\|-1|,\left|x^{*}(y)-1\right|\right\} \\
& \geq \beta_{X}(\tilde{x}, \varepsilon)-\frac{\delta}{1-\tau},
\end{aligned}
$$

and since $0<\tau<1$ was arbitrary, it follows that

$$
\max \left\{1-\left\|\frac{x+y}{2}\right\|,|\|y\|-1|,\left|x^{*}(y)-1\right|\right\} \geq \beta_{X}(\tilde{x}, \varepsilon)-\delta .
$$

Again, since $\left(y, x^{*}\right) \in V_{x, \varepsilon}$ was arbitrary we can conclude that

$$
\beta_{X}(\tilde{x}, \varepsilon)-\beta_{X}(x, \varepsilon) \leq \delta=\|x-\tilde{x}\|
$$

and by symmetry it follows that

$$
\left|\beta_{X}(\tilde{x}, \varepsilon)-\beta_{X}(x, \varepsilon)\right| \leq\|x-\tilde{x}\| .
$$

Analogously one can prove that

$$
\left|\beta_{X}(x, \widetilde{\mathcal{\varepsilon}})-\beta_{X}(x, \varepsilon)\right| \leq|\varepsilon-\widetilde{\varepsilon}|
$$

for all $x \in S_{X}$ and all $0<\varepsilon, \widetilde{\mathcal{E}}, \leq 2$. An application of the triangle inequality then yields the result. 
In the paper [16] Kamińska and Turett proved various theorems concerning different rotundity properties of KötheBochner spaces. For example, by [16, Theorem 5] if $E$ has the so-called Fatou property and is LUR, then $E(X)$ is LUR whenever $X$ is LUR. We will adopt the technique of proof from [16, Theorem 5] to show the following result.

Theorem 15. If $E$ is $L U R$ and $X$ is sluacs smooth, then $E(X)$ is also sluacs.

Proof. Since $E$ is LUR it is order continuous.

Let $0<\varepsilon \leq 2$ and $f \in S_{E(X)}$ be arbitrary and let

$$
A_{n}:=\left\{t \in S: f(t) \neq 0, \beta_{X}\left(\frac{f(t)}{\|f(t)\|}, \frac{\varepsilon}{8}\right) \geq \frac{1}{n}\right\}
$$

for every $n \in \mathbb{N}$. Since by the previous lemma $\beta_{X}(\cdot, \varepsilon / 8)$ is continuous, it follows that the sets $A_{n}$ are measurable. Also, the sequence $\left(A_{n}\right)_{n \in \mathbb{N}}$ is increasing and because $X$ is sluacs, we have $\bigcup_{n=1}^{\infty} A_{n}=\{t \in S: f(t) \neq 0\}$; hence $\left(\|f(\cdot)\| \chi_{S \backslash A_{n}}\right)_{n \in \mathbb{N}}$ decreases pointwise to zero. The order continuity of $E$ implies \|\|$f(\cdot)\left\|\chi_{S \backslash A_{n}}\right\|_{E} \rightarrow 0$, and thus we can find $n_{0} \in \mathbb{N}$ with

$$
\|\| f(\cdot)\left\|\chi_{S \backslash A_{n_{0}}}\right\|_{E} \leq \frac{\varepsilon}{64} \text {. }
$$

Now let us take $g \in S_{E(X)}$ and $l \in S_{E(X)^{*}}$ with $l(g)=1$ and $l(f) \leq 1-\varepsilon$. Let $l$ be represented by $[F] \in E^{\prime}\left(X^{*}, w^{*}\right)$. As in the proof of Proposition 9 we can conclude that

$$
\begin{gathered}
\int_{S}\|F(t)\|\|g(t)\| \mathrm{d} \mu(t)=1, \\
\|F(t)\|\|g(t)\|=F(t)(g(t)) \quad \text { a.e. }
\end{gathered}
$$

Next we define

$$
\begin{gathered}
C:=\{t \in S: F(t) \neq 0\}, \\
B:=\{t \in C: F(t)(g(t)-f(t)) \\
\left.\geq \frac{\varepsilon}{4}\|F(t)\| \max \{\|f(t)\|,\|g(t)\|\}\right\} .
\end{gathered}
$$

Then $B$ is measurable, and

$$
\begin{array}{rl}
\int_{S \backslash B} & F(t)(g(t)-f(t)) \mathrm{d} \mu(t) \\
& \leq \frac{\varepsilon}{4} \int_{S \backslash B}\|F(t)\| \max \{\|f(t)\|,\|g(t)\|\} \mathrm{d} \mu(t) \\
& \leq \frac{\varepsilon}{4} \int_{S \backslash B}\|F(t)\|(\|f(t)\|+\|g(t)\|) \mathrm{d} \mu(t) \leq \frac{\varepsilon}{4} 2=\frac{\varepsilon}{2} .
\end{array}
$$

Since $l(g-f) \geq \varepsilon$, it follows that

$$
\int_{B} F(t)(g(t)-f(t)) \mathrm{d} \mu(t) \geq \frac{\varepsilon}{2} .
$$

Let us fix $0<\eta<\min \left\{\varepsilon / 16,1 / 2 n_{0}\right\}$ such that

$$
\frac{\eta}{1-\eta}<\frac{2}{n_{0}}
$$

Now consider the following sets:

$$
\begin{aligned}
& B_{1}:=\{t \in B:\|g(t)\|<(1-\eta)\|f(t)\|\}, \\
& B_{2}:=\{t \in B:(1-\eta)\|f(t)\| \leq\|g(t)\| \leq\|f(t)\|\}, \\
& B_{3}:=\{t \in B:(1-\eta)\|g(t)\| \leq\|f(t)\|<\|g(t)\|\}, \\
& B_{4}:=\{t \in B:(1-\eta)\|g(t)\|>\|f(t)\|\} .
\end{aligned}
$$

Then $B_{1}, \ldots, B_{4}$ are measurable, pairwise disjoint, and $\bigcup_{i=1}^{4} B_{i}=B$. Thus by (96) there exists some $i \in\{1, \ldots, 4\}$ such that

$$
\int_{B_{i}} F(t)(g(t)-f(t)) \mathrm{d} \mu(t) \geq \frac{\varepsilon}{8} .
$$

If $i=1$, then, since $\|g(t)\| \leq\|f(t)\|$ for $t \in B_{1}$, it follows that

$$
\int_{B_{1}}\|F(t)\|\|f(t)\| \mathrm{d} \mu(t) \geq \frac{\varepsilon}{16},
$$

and again by definition of $B_{1}$ we obtain

$$
\begin{aligned}
& \|\| g(\cdot)\|-\| f(\cdot)\|\|_{E} \\
& \quad=\|\| g(\cdot)\|-\| f(\cdot)\|\|_{E} \\
& \quad \geq \int_{B_{1}}\|F(t)\|(\|f(t)\|-\|g(t)\|) \mathrm{d} \mu(t) \\
& \quad \geq \eta \int_{B_{1}}\|F(t)\|\|f(t)\| \mathrm{d} \mu(t) \geq \eta \frac{\varepsilon}{16}
\end{aligned}
$$

and hence

$$
\left\|\frac{f+g}{2}\right\|_{E(X)} \leq\left\|\frac{\|f(\cdot)\|+\|g(\cdot)\|}{2}\right\|_{E} \leq 1-\delta_{E}\left(\|f(\cdot)\|, \eta \frac{\varepsilon}{16}\right) .
$$

In the case of $i=4$ one can obtain the same statement by an analogous argument. To treat the remaining cases we need some preliminary considerations.

Let us denote by $N$ the null set on which the equality from (93) does not hold and suppose that $t \in B_{2} \cap A_{n_{0}} \cap(S \backslash N)$. Then in particular $t \in B$ and $\|f(t)\| \geq\|g(t)\|$, and hence

$$
\frac{F(t)}{\|F(t)\|}\left(\frac{g(t)}{\|f(t)\|}-\frac{f(t)}{\|f(t)\|}\right) \geq \frac{\varepsilon}{4} .
$$

Moreover, by the definitions of $B_{2}$ and $A_{n_{0}}$ and the choice of $\eta$ we have

$$
\begin{aligned}
\left|\| \frac{g(t)}{\|f(t)\|}\right|-1 \mid & =\left|\frac{\|g(t)\|}{\|f(t)\|}-1\right| \leq \eta<\frac{1}{n_{0}} \\
& \leq \beta_{X}\left(\frac{f(t)}{\|f(t)\|}, \frac{\varepsilon}{8}\right) \leq \beta_{X}\left(\frac{f(t)}{\|f(t)\|}, \frac{\varepsilon}{4}\right) .
\end{aligned}
$$

Since $t \in(S \backslash N)$, we also have

$$
\left|\frac{F(t)}{\|F(t)\|}\left(\frac{g(t)}{\|f(t)\|}\right)-1\right|=\left|\frac{\|g(t)\|}{\|f(t)\|}-1\right|<\beta_{X}\left(\frac{f(t)}{\|f(t)\|}, \frac{\varepsilon}{4}\right) .
$$


So by definition of $\beta_{X}$ we must have

$$
\frac{1}{2}\left\|\frac{f(t)}{\|f(t)\|}+\frac{g(t)}{\|f(t)\|}\right\| \leq 1-\beta_{X}\left(\frac{f(t)}{\|f(t)\|}, \frac{\varepsilon}{4}\right) \leq 1-\frac{1}{n_{0}} .
$$

Once more by the definition of $B_{1}$ this implies

$$
\begin{aligned}
\left\|\frac{f(t)+g(t)}{2}\right\| & \leq\left(1-\frac{1}{n_{0}}\right)\|f(t)\| \\
& \leq \frac{1-1 / n_{0}}{2(1-\eta)}(\|f(t)\|+\|g(t)\|) \\
& =\frac{1}{2}\left(1-\alpha_{1}\right)(\|f(t)\|+\|g(t)\|),
\end{aligned}
$$

where $\alpha_{1}:=\left(1 / n_{0}-\eta\right)(1-\eta)^{-1}>0$.

Now suppose that $t \in B_{3} \cap A_{n_{0}} \cap(S \backslash N)$. Then

$$
\frac{F(t)}{\|F(t)\|}\left(\frac{g(t)}{\|g(t)\|}-\frac{f(t)}{\|g(t)\|}\right) \geq \frac{\varepsilon}{4} .
$$

Consequently

$$
\begin{aligned}
& \frac{F(t)}{\|F(t)\|}\left(\frac{g(t)}{\|g(t)\|}-\frac{f(t)}{\|f(t)\|}\right) \\
& \quad \geq \frac{\varepsilon}{4}+\frac{F(t)}{\|F(t)\|}\left(\frac{f(t)}{\|g(t)\|}-\frac{f(t)}{\|f(t)\|}\right) \\
& \quad \geq \frac{\varepsilon}{4}-\left\|\frac{f(t)}{\|g(t)\|}-\frac{f(t)}{\|f(t)\|}\right\|=\frac{\varepsilon}{4}-\left|\frac{\|f(t)\|}{\|g(t)\|}-1\right| \\
& \geq \frac{\varepsilon}{4}-\eta \geq \frac{\varepsilon}{8} .
\end{aligned}
$$

Since $\|F(t)\|\|g(t)\|=F(t)(g(t))$, the definition of $\beta_{X}$ implies that

$$
\frac{1}{2}\left\|\frac{f(t)}{\|f(t)\|}+\frac{g(t)}{\|g(t)\|}\right\| \leq 1-\beta_{X}\left(\frac{f(t)}{\|f(t)\|}, \frac{\varepsilon}{8}\right) \leq 1-\frac{1}{n_{0}},
$$

where the latter inequality holds because of $t \in A_{n_{0}}$. It follows that

$$
\begin{aligned}
& \frac{1}{2}\left\|\frac{f(t)}{\|f(t)\|}+\frac{g(t)}{\|f(t)\|}\right\| \\
& \leq 1-\frac{1}{n_{0}}+\frac{1}{2}\left\|\frac{g(t)}{\|f(t)\|}-\frac{g(t)}{\|g(t)\|}\right\| \\
& \quad=1-\frac{1}{n_{0}}+\frac{1}{2}\left|\frac{\|g(t)\|}{\|f(t)\|}-1\right| \\
& \leq 1-\frac{1}{n_{0}}+\frac{1}{2}\left(\frac{1}{1-\eta}-1\right)=1-\alpha_{2},
\end{aligned}
$$

where $\alpha_{2}:=1 / n_{0}-\eta(2-2 \eta)^{-1}$ which by (97) is greater than zero. Because of $\|f(t)\| \leq\|g(t)\|$, it follows that

$$
\|f(t)+g(t)\| \leq\left(1-\alpha_{2}\right)(\|f(t)\|+\|g(t)\|) .
$$

So if we put $\alpha=\min \left\{\alpha_{1}, \alpha_{2}\right\}$ and $P=B_{2} \cap A_{n_{0}} \cap(S \backslash N)$, $Q=B_{3} \cap A_{n_{0}} \cap(S \backslash N)$ then

$$
\|f(t)+g(t)\| \leq(1-\alpha)(\|f(t)\|+\|g(t)\|), \quad \forall t \in P \cup Q .
$$

Now we will show that if $i=2$ and $i=3$, respectively then

$$
\begin{aligned}
\int_{P}\|F(t)\|\|f(t)\| \mathrm{d} \mu(t) \\
\quad \geq \frac{\varepsilon}{64} \text { resp. } \int_{Q}\|F(t)\|\|f(t)\| \mathrm{d} \mu(t) \geq \frac{\varepsilon}{64} .
\end{aligned}
$$

Let us first assume that $i=2$, that is,

$$
\int_{B_{2}} F(t)(g(t)-f(t)) \mathrm{d} \mu(t) \geq \frac{\varepsilon}{8} .
$$

Since $\|f(t)\| \geq\|g(t)\|$ for $t \in B_{2}$ it follows that

$$
\int_{B_{2}}\|F(t)\|\|f(t)\| \mathrm{d} \mu(t) \geq \frac{\varepsilon}{16} .
$$

Because $N$ is a null set, we have

$$
\begin{aligned}
& \int_{P}\|F(t)\|\|f(t)\| \mathrm{d} \mu(t) \\
&=\int_{B_{2} \cap A_{n_{0}}}\|F(t)\|\|f(t)\| \mathrm{d} \mu(t) \\
&=\int_{B_{2}}\|F(t)\|\|f(t)\| \mathrm{d} \mu(t) \\
& \quad-\int_{B_{2} \backslash A_{n_{0}}}\|F(t)\|\|f(t)\| \mathrm{d} \mu(t) \\
& \geq \frac{\varepsilon}{16}-\int_{S \backslash A_{n_{0}}}\|F(t)\|\|f(t)\| \mathrm{d} \mu(t) \\
& \geq \frac{\varepsilon}{16}-\|\| f(\cdot)\left\|\chi_{S \backslash A_{n_{0}}}\right\|_{E} \geq \frac{\varepsilon}{16}-\frac{\varepsilon}{64} \geq \frac{\varepsilon}{64},
\end{aligned}
$$

where the second last inequality holds because of (91).

Now assume that $i=3$; that is,

$$
\int_{B_{3}} F(t)(g(t)-f(t)) \mathrm{d} \mu(t) \geq \frac{\varepsilon}{8} .
$$

It follows that

$$
\begin{aligned}
\frac{\varepsilon}{8} & \leq \int_{B_{3}}\|F(t)\|(\|g(t)\|+\|f(t)\|) \mathrm{d} \mu(t) \\
& \leq \int_{B_{3}}\|F(t)\|\left(1+\frac{1}{1-\eta}\right)\|f(t)\| \mathrm{d} \mu(t) \\
& \leq 4 \int_{B_{3}}\|F(t)\|\|f(t)\| \mathrm{d} \mu(t),
\end{aligned}
$$

and hence as before we get

$$
\int_{Q}\|F(t)\|\|f(t)\| \mathrm{d} \mu(t) \geq \frac{\varepsilon}{32}-\frac{\varepsilon}{64}=\frac{\varepsilon}{64} .
$$


So if $i=2$, or $i=3$ then there is $R \in\{P, Q\}$ such that

$$
\|\| f(\cdot)\left\|\chi_{R}\right\|_{E} \geq \int_{R}\|F(t)\|\|f(t)\| \mathrm{d} \mu(t) \geq \frac{\varepsilon}{64} .
$$

Put $h=\|f(\cdot)\|\left(1-2 \alpha \chi_{R}\right)$. Then $h \in B_{E}$, and moreover \|\|$f(\cdot)\|-h\|_{E}=2 \alpha\|\| f(\cdot)\left\|\chi_{R}\right\|_{E} \geq \alpha \varepsilon / 32$; hence

$$
\begin{aligned}
\|\| f(\cdot)\left\|\left(1-\alpha \chi_{R}\right)\right\|_{E} & =\frac{1}{2}\|\| f(\cdot)\|+h\|_{E} \\
& \leq 1-\delta_{E}\left(\|f(\cdot)\|, \frac{\varepsilon \alpha}{32}\right) .
\end{aligned}
$$

We further have

$$
\begin{aligned}
& \left\|\frac{f+g}{2}\right\|_{E(X)} \\
& \quad \leq \frac{1}{2}\left\|(\|f(\cdot)\|+\|g(\cdot)\|) \chi_{S \backslash R}+\right\| f(\cdot)+g(\cdot)\left\|\chi_{R}\right\|_{E} \\
& \quad \stackrel{(113)}{\leq} \frac{1}{2} \|(\|f(\cdot)\|+\|g(\cdot)\|) \chi_{S \backslash R} \\
& \quad \leq \frac{1}{2}\|\| g(\cdot)\|+\| f(\cdot)\|-\alpha\| f(\cdot)\left\|\chi_{R}\right\|_{E} \\
& \leq \frac{1}{2}+\frac{1}{2}\|\| f(\cdot)\left\|\left(1-\alpha \chi_{R}\right)\right\|_{E} \\
& \leq \frac{1}{2}+\frac{1}{2}\left(1-\delta_{E}\left(\|f(\cdot)\|, \frac{\varepsilon \alpha}{32}\right)\right) \\
& =1-\frac{1}{2} \delta_{E}\left(\|f(\cdot)\|, \frac{\varepsilon \alpha}{32}\right) .
\end{aligned}
$$

Altogether we have shown that, for

$$
\delta:=\min \left\{\frac{1}{2} \delta_{E}\left(\|f(\cdot)\|, \frac{\varepsilon \alpha}{32}\right), \delta_{E}\left(\|f(\cdot)\|, \frac{\varepsilon \eta}{16}\right)\right\}>0,
$$

we have for every $g \in S_{E(X)}$ and every $l \in S_{E(X)^{*}}$ with $l(g)=1$ and $l(f) \leq 1-\varepsilon$

$$
\left\|\frac{f+g}{2}\right\|_{E(X)} \leq 1-\delta .
$$

By the aforementioned characterisation of sluacs spaces ([4, Proposition 2.1]), this implies that $E(X)$ is sluacs.

Next we will have a look at the case of wuacs spaces.

Theorem 16. If $\mu$ is a $\sigma$-finite measure and $E$ is wuacs, reflexive, and has the Kadets-Klee property, then $E(X)$ is wuacs whenever $X$ is wuacs.

Proof. Note that since $E$ is reflexive (or since it has the KadetsKlee property), it is order continuous.

Let us take two sequences $\left(f_{n}\right)_{n \in \mathbb{N}}$ and $\left(g_{n}\right)_{n \in \mathbb{N}}$ in the unit sphere of $E(X)$ such that $\left\|f_{n}+g_{n}\right\|_{E(X)} \rightarrow 2$ and a functional $l \in S_{E(X)^{*}}$, as usual represented by $[F] \in E^{\prime}\left(X^{*}, w^{*}\right)$, with $l\left(f_{n}\right) \rightarrow 1$.

As in the proof of Theorem 10 we find

$$
\lim _{n \rightarrow \infty} \int_{S}\|F(t)\|\left\|f_{n}(t)\right\| \mathrm{d} \mu(t)=1,
$$

and by passing to a subsequence also

$$
\lim _{n \rightarrow \infty}\left(\|F(t)\|\left\|f_{n}(t)\right\|-F(t)\left(f_{n}(t)\right)\right)=0 \quad \text { a.e. }
$$

It is also easy to see that

$$
\begin{gathered}
\lim _{n \rightarrow \infty}\|\| f_{n}(\cdot)\|+\| g_{n}(\cdot)\|\|_{E}=2, \\
\lim _{n \rightarrow \infty}\|\| f_{n}(\cdot)\|+\| g_{n}(\cdot)\|+\| f_{n}(\cdot)+g_{n}(\cdot)\|\|_{E}=4 .
\end{gathered}
$$

Since $E$ is wuacs, it follows from (126) and (128) that

$$
\lim _{n \rightarrow \infty} \int_{S}\|F(t)\|\left\|g_{n}(t)\right\| \mathrm{d} \mu(t)=1 .
$$

Again since $E$ is wuacs and because of (126), (128), (129), and (130) we can deduce that

$$
\begin{aligned}
& \lim _{n \rightarrow \infty} \int_{S}\|F(t)\|\left(\left\|f_{n}(t)\right\|+\left\|g_{n}(t)\right\|-\left\|f_{n}(t)+g_{n}(t)\right\|\right) \mathrm{d} \mu(t) \\
& \quad=0 .
\end{aligned}
$$

Hence we can pass to a further subsequence such that

$$
\lim _{n \rightarrow \infty}\|F(t)\|\left(\left\|f_{n}(t)\right\|+\left\|g_{n}(t)\right\|-\left\|f_{n}(t)+g_{n}(t)\right\|\right)=0 \quad \text { a.e. }
$$

By the reflexivity of $E$ we can pass once more to a subsequence such that $\left(\left\|f_{n}(\cdot)\right\|\right)_{n \in \mathbb{N}}$ and $\left(\left\|g_{n}(\cdot)\right\|\right)_{n \in \mathbb{N}}$ are weakly convergent to $h_{1} \in B_{E}$ and $h_{2} \in B_{E}$, respectively. In view of (126) and (130) it follows that

$$
\int_{S}\|F(t)\| h_{i}(t) \mathrm{d} \mu(t)=1 \quad \forall i \in\{1,2\}
$$

hence $\left\|h_{1}\right\|_{E}=\left\|h_{2}\right\|_{E}=1$, and moreover

$$
\left\|h_{1}+h_{2}\right\|_{E}=2 \text {. }
$$

The fact that $E$ has the Kadets-Klee property implies that

$$
\|\| f_{n}(\cdot)\left\|-h_{1}\right\|_{E} \longrightarrow 0, \quad\|\| g_{n}(\cdot)\left\|-h_{2}\right\|_{E} \longrightarrow 0,
$$

and thus by Lemma 7 we can, for the last time, pass to a subsequence such that

$$
\lim _{n \rightarrow \infty}\left\|f_{n}(t)\right\|=h_{1}(t), \quad \lim _{n \rightarrow \infty}\left\|g_{n}(t)\right\|=h_{2}(t) \quad \text { a.e. }
$$

Let $N_{1}, N_{2}$, and $N_{3}$ denote the null sets on which the convergence statement from (127), (132), and (136), respectively, does not hold and put $N=N_{1} \cup N_{2} \cup N_{3}$ as well as $B=\{t \in$ $S \backslash N: F(t) \neq 0$ and $\left.h_{2}(t) \neq 0\right\}$ and $C=\left\{t \in B: h_{1}(t)=0\right\}$. 
Because of (134) and since $E$ is in particular acs, we can show just as in the proof of Proposition 9 that $C$ is a null set.

The fact that $X$ is wuacs together with Lemma 5 easily implies that

$$
\begin{array}{r}
\lim _{n \rightarrow \infty}\left(\|F(t)\|\left\|g_{n}(t)\right\|-F(t)\left(g_{n}(t)\right)\right)=0, \\
\forall t \in S \backslash(N \cup C) .
\end{array}
$$

By the weak convergence of $\left(\left\|g_{n}(\cdot)\right\|\right)_{n \in \mathbb{N}}$ to $h_{2}$ we have

$$
\begin{aligned}
\lim _{n \rightarrow \infty} \int_{A}\|F(t)\|\left\|g_{n}(t)\right\| \mathrm{d} \mu(t) \\
=\int_{A}\|F(t)\| h_{2}(t) \mathrm{d} \mu(t) \quad \forall A \in \mathscr{A} .
\end{aligned}
$$

Since $E$ is reflexive, $E^{\prime}$ is order continuous, and thus we can deduce as in the proof of Theorem 13, with the aid of Vitali's lemma, (138), (137), and the fact that $N \cup C$ is a null set, that

$$
\lim _{n \rightarrow \infty} \int_{S}\left(\|F(t)\|\left\|g_{n}(t)\right\|-F(t)\left(g_{n}(t)\right)\right) \mathrm{d} \mu(t)=0 .
$$

Because of (130), it follows that

$$
\lim _{n \rightarrow \infty} l\left(g_{n}\right)=\lim _{n \rightarrow \infty} \int_{S} F(t)\left(g_{n}(t)\right) \mathrm{d} \mu(t)=1,
$$

and we are done.

If we combine the techniques of the proofs of Theorems 16 and 13 , we can also obtain another result concerning luacs ${ }^{+}$ spaces (we omit the details).

Theorem 17. If $\mu$ is a $\sigma$-finite measure and $E$ is luacs ${ }^{+}$, reflexive and has the Kadets-Klee property, then $E(X)$ is luacs ${ }^{+}$ whenever $X$ is luacs ${ }^{+}$.

It is further possible to obtain another sufficient condition for $E(X)$ to be sluacs.

Theorem 18. If $\mu$ is a $\sigma$-finite measure and $E$ is sluacs ${ }^{+}$and reflexive and both $E$ and $E^{*}$ have the Kadets-Klee property, then $E(X)$ is sluacs whenever $X$ is sluacs.

Proof. Let $\left(f_{n}\right)_{n \in \mathbb{N}}$ be a sequence in $S_{E(X)}$ and $f \in S_{E(X)}$ such that we have $\left\|f_{n}+f\right\|_{E(X)} \rightarrow 2$. Also, let $\left(l_{n}\right)_{n \in \mathbb{N}}$ be a sequence in $S_{E(X)^{*}}$ such that $l_{n}\left(f_{n}\right) \rightarrow 1$. If we represent each $l_{n}$ by $\left[F_{n}\right] \in E^{\prime}\left(X^{*}, w^{*}\right)$, we can obtain as usual

$$
\lim _{n \rightarrow \infty} \int_{S}\left\|F_{n}(t)\right\|\left\|f_{n}(t)\right\| \mathrm{d} \mu(t)=1
$$

and by passing to a subsequence also

$$
\lim _{n \rightarrow \infty}\left(\left\|F_{n}(t)\right\|\left\|f_{n}(t)\right\|-F_{n}(t)\left(f_{n}(t)\right)\right)=0 \quad \text { a.e. }
$$

as well as

$$
\begin{gathered}
\lim _{n \rightarrow \infty}\|\| f_{n}(\cdot)\|+\| f(\cdot)\|\|_{E}=2, \\
\lim _{n \rightarrow \infty}\|\| f_{n}(\cdot)+f(\cdot)\|+\| f_{n}(\cdot)\|+\| f(\cdot)\|\|_{E}=4, \\
\lim _{n \rightarrow \infty}\|\| f_{n}(\cdot)+f(\cdot)\|+\| f_{n}(\cdot)\|\|_{E}=3, \\
\lim _{n \rightarrow \infty}\|\| f_{n}(\cdot)+f(\cdot)\|+\| f(\cdot)\|\|_{E}=3, \\
\lim _{n \rightarrow \infty}\|\| f_{n}(\cdot)+f(\cdot)\|+\| f_{n}(\cdot)\|+3\| f(\cdot)\|\|_{E}=6 .
\end{gathered}
$$

Using the fact that $E$ is sluacs ${ }^{+}$we can conclude that

$$
\begin{aligned}
& \lim _{n \rightarrow \infty} \int_{S}\left\|F_{n}(t)\right\|\|f(t)\| \mathrm{d} \mu(t)=1 \\
& \lim _{n \rightarrow \infty} \int_{S}\left\|F_{n}(t)\right\|\left(\left\|f_{n}(t)\right\|+\|f(t)\|-\left\|f_{n}(t)+f(t)\right\|\right) \mathrm{d} \mu(t) \\
& =0 .
\end{aligned}
$$

So we can pass to another subsequence such that

$$
\lim _{n \rightarrow \infty}\left\|F_{n}(t)\right\|\left(\left\|f_{n}(t)\right\|+\|f(t)\|-\left\|f_{n}(t)+f(t)\right\|\right)=0 \quad \text { a.e. }
$$

Since $E$ (and hence also $E^{*}$ ) is reflexive, we may assume without loss of generality that $\left(\left\|f_{n}(\cdot)\right\|\right)_{n \in \mathbb{N}}$ is weakly convergent to some $h \in B_{E}$ and that $\left(\left\|F_{n}(\cdot)\right\|\right)_{n \in \mathbb{N}}$ is weakly convergent to some $g \in B_{E^{*}}=B_{E^{\prime}}$.

It follows from (148) that

$$
\int_{S} g(t)\|f(t)\| \mathrm{d} \mu(t)=1
$$

and hence $g \in S_{E^{*}}$. Because of (151), (143), and the fact that $E$ is sluacs ${ }^{+}$, we get that

$$
\lim _{n \rightarrow \infty} \int_{S} g(t)\left\|f_{n}(t)\right\| \mathrm{d} \mu(t)=1
$$

and consequently

$$
\int_{S} g(t) h(t) \mathrm{d} \mu(t)=1
$$

whence $h \in S_{E}$. Since both $E$ and $E^{*}$ have the Kadets-Klee property, it follows that

$$
\|\| f_{n}(\cdot)\|-h\|_{E} \longrightarrow 0, \quad\|\| F_{n}(\cdot)\|-g\|_{E^{\prime}} \longrightarrow 0 .
$$

Thus we can pass once more to a subsequence such that

$$
\lim _{n \rightarrow \infty}\left\|f_{n}(t)\right\|=h(t), \quad \lim _{n \rightarrow \infty}\left\|F_{n}(t)\right\|=g(t) \quad \text { a.e. }
$$

Combining (153) and (151) we also obtain

$$
\|h+\| f(\cdot)\|\|_{E}=2 .
$$


Let $N$ be a null set such that the convergence statements of (142), (150), and (155) hold for every $t \in S \backslash N$, and put $B=\{t \in$ $S \backslash N: g(t) \neq 0$ and $f(t) \neq 0\}$ as well as $C=\{t \in B: h(t)=0\}$.

Similar to the arguments in the proof of Theorem 16 one can see that $C$ is a null set and then, using the fact that $X$ is sluacs, deduce that

$$
\lim _{n \rightarrow \infty}\left(\left\|F_{n}(t)\right\|\|f(t)\|-F_{n}(t)(f(t))\right)=0 \quad \text { a.e. }
$$

By our usual method based on Vitali's lemma we can conclude that for every $A \in \mathscr{A}$ with $\mu(A)<\infty$ we have

$$
\lim _{n \rightarrow \infty} \int_{A}\left(\left\|F_{n}(t)\right\|\|f(t)\|-F_{n}(t)(f(t))\right) \mathrm{d} \mu(t)=0 .
$$

Now we fix an increasing sequence $\left(A_{m}\right)_{m \in \mathbb{N}}$ in $\mathscr{A}$ such that $\mu\left(A_{m}\right)<\infty$ for all $m \in \mathbb{N}$ and $\bigcup_{m=1}^{\infty} A_{m}=S$. The order continuity of $E$ implies \|\|$f(\cdot)\left\|\chi_{S \backslash A_{m}}\right\|_{E} \rightarrow 0$. Analogous to the argument at the end of the proof of Theorem 13, this together with (158) leads to

$$
\lim _{n \rightarrow \infty} \int_{S}\left(\left\|F_{n}(t)\right\|\|f(t)\|-F_{n}(t)(f(t))\right) \mathrm{d} \mu(t)=0 .
$$

Taking into account (148) we arrive at

$$
\lim _{n \rightarrow \infty} l_{n}(f)=\lim _{n \rightarrow \infty} \int_{S} F_{n}(t)(f(t)) \mathrm{d} \mu(t)=1,
$$

and the proof is finished.

Next we will consider sufficient conditions for a KötheBochner space to be sluacs ${ }^{+}$(recall that a dual Banach space $X^{*}$ is said to have the Kadets-Klee* property if it fulfils the definition of the Kadets-Klee property with weak-replaced by weak $^{*}$-convergence).

Theorem 19. Let $E$ be a Köthe function space over the complete $\sigma$-finite measure space $(S, \mathcal{A}, \mu)$, and let $X$ be an sluacs ${ }^{+}$ Banach space. If $E^{*}$ has the Kadets-Klee* property and in addition

(a) E is sluacs ${ }^{+}$, reflexive, and has the Kadets-Klee property or

(b) $E$ is LUR and $B_{E^{*}}$ is weak*-sequentially compact, then $E(X)$ is sluacs ${ }^{+}$.

Proof. By Theorems 15 and 18 we already know that $E(X)$ is in both cases sluacs. Note also that in both cases $E$ is order continuous. Now take a sequence $\left(f_{n}\right)_{n \in \mathbb{N}}$ in $S_{E(X)}$ and $f \in S_{E(X)}$ such that $\left\|f_{n}+f\right\|_{E(X)} \rightarrow 2$, and let $\left(l_{n}\right)_{n \in \mathbb{N}}$ be a sequence in $S_{E(X)^{*}}$ such that $l_{n}(f) \rightarrow 1$. If we represent each $l_{n}$ by $\left[F_{n}\right] \in E^{\prime}\left(X^{*}, w^{*}\right)$ we can obtain as usual

$$
\lim _{n \rightarrow \infty} \int_{S}\left\|F_{n}(t)\right\|\|f(t)\| \mathrm{d} \mu(t)=1
$$

and by passing to a subsequence also

$$
\lim _{n \rightarrow \infty}\left(\left\|F_{n}(t)\right\|\|f(t)\|-F_{n}(t)(f(t))\right)=0 \text { a.e. }
$$

as well as

$$
\begin{gathered}
\lim _{n \rightarrow \infty}\|\| f_{n}(\cdot)\|+\| f(\cdot)\|\|_{E}=2, \\
\lim _{n \rightarrow \infty}\|\| f_{n}(\cdot)+f(\cdot)\|+\| f_{n}(\cdot)\|+\| f(\cdot)\|\|_{E}=4, \\
\lim _{n \rightarrow \infty}\|\| f_{n}(\cdot)+f(\cdot)\|+\| f_{n}(\cdot)\|\|_{E}=3, \\
\lim _{n \rightarrow \infty}\|\| f_{n}(\cdot)+f(\cdot)\|+\| f(\cdot)\|\|_{E}=3, \\
\lim _{n \rightarrow \infty}\|\| f_{n}(\cdot)+f(\cdot)\|+\| f_{n}(\cdot)\|+3\| f(\cdot)\|\|_{E}=6 .
\end{gathered}
$$

Since $E$ is sluacs ${ }^{+}$, it follows that

$$
\begin{gathered}
\lim _{n \rightarrow \infty} \int_{S}\left\|F_{n}(t)\right\|\left\|f_{n}(t)\right\| \mathrm{d} \mu(t)=1, \\
\lim _{n \rightarrow \infty} \int_{S}\left\|F_{n}(t)\right\|\left(\left\|f_{n}(t)\right\|+\|f(t)\|\right. \\
\left.-\left\|f_{n}(t)+f(t)\right\|\right) \mathrm{d} \mu(t)=0,
\end{gathered}
$$

so that by passing to another subsequence we can assume

$$
\lim _{n \rightarrow \infty}\left\|F_{n}(t)\right\|\left(\left\|f_{n}(t)\right\|+\|f(t)\|-\left\|f_{n}(t)+f(t)\right\|\right)=0 \quad \text { a.e. }
$$

In both cases (a) and (b) the dual unit ball $B_{E^{*}}$ is weak* sequentially compact, so that we can also assume the weak ${ }^{*}$ convergence of $\left(\left\|F_{n}(\cdot)\right\|\right)_{n \in \mathbb{N}}$ to some $g \in B_{E^{*}}$. It follows from (161) that

$$
\int_{S} g(t)\|f(t)\| \mathrm{d} \mu(t)=1
$$

and hence $\|g\|_{E^{\prime}}=1$. Since $E^{*}$ has the Kadets-Klee* property, we get

$$
\|\| F_{n}(\cdot)\|-g\|_{E^{\prime}} \longrightarrow 0
$$

and thus we can, by passing to yet another subsequence, assume that

$$
\lim _{n \rightarrow \infty}\left\|F_{n}(t)\right\|=g(t) \quad \text { a.e. }
$$

Next we claim that there is an $h \in S_{E}$ such that

$$
\int_{S} g(t) h(t) \mathrm{d} \mu(t)=1
$$

and, after passing to a subsequence once more,

$$
\|\| f_{n}(\cdot)\|-h\|_{E} \longrightarrow 0 .
$$

In case (b) $E$ is LUR, and thus by (163) and (171) we can take $h=\|f(\cdot)\|$. In case (a) $E$ is reflexive, and hence we can assume that $\left(\left\|f_{n}(\cdot)\right\|\right)_{n \in \mathbb{N}}$ is weakly convergent to some $h \in B_{E}$. Then (174) follows from (172) and (168). This also implies $\|h\|_{E}=1$, and by the Kadets-Klee property of $E$ we have (175).

By (175) we may assume that

$$
\lim _{h n \rightarrow \infty}\left\|f_{n}(t)\right\|=h(t) \quad \text { a.e. }
$$


Note that (171) and (174) imply that \|\|$f(\cdot)\|+h\|_{E}=2$. Using all this and the fact that $X$ is sluacs ${ }^{+}$one can first prove, analogously to the arguments in the proof of Theorem 18, that

$$
\lim _{n \rightarrow \infty}\left(\left\|F_{n}(t)\right\|\left\|f_{n}(t)\right\|-F_{n}(t)\left(f_{n}(t)\right)\right)=0 \quad \text { a.e. }
$$

and then

$$
\lim _{n \rightarrow \infty} \int_{A}\left(\left\|F_{n}(t)\right\|\left\|f_{n}(t)\right\|-F_{n}(t)\left(f_{n}(t)\right)\right) \mathrm{d} \mu(t)=0
$$

for every $A \in \mathscr{A}$ with $\mu(A)<\infty$.

Let us now fix a sequence $\left(A_{m}\right)_{m \in \mathbb{N}}$ in $\mathscr{A}$ as in the proof of Theorem 18. The order continuity of $E$ implies \|\|$f(\cdot)\left\|\chi_{S \backslash A_{m}}\right\|_{E} \rightarrow 0$.

Let $\varepsilon>0$ be arbitrary. Since $E$ is sluacs ${ }^{+}$there exists a $\delta>$ 0 such that for all $b \in S_{E}$ and all $l \in B_{E^{*}}$ with $\|b+\| f(\cdot)\|\|_{E} \geq$ $2(1-\delta)$ and $l(\|f(\cdot)\|) \geq 1-\delta$ one has $l(b) \geq 1-\varepsilon$.

Fix $m_{0} \in \mathbb{N}$ with \|\|$f(\cdot)\left\|\chi_{S \backslash A_{m_{0}}}\right\|_{E} \leq \delta / 2$. Because of (163), (161) and (178) there is an $N \in \mathbb{N}$ such that for all $n \geq N$ the inequalities

$$
\begin{gathered}
\|\| f_{n}(\cdot)\|+\| f(\cdot)\|\|_{E} \geq 2(1-\delta), \\
\int_{S}\left\|F_{n}(t)\right\|\|f(t)\| \mathrm{d} \mu(t) \geq 1-\frac{\delta}{2}, \\
\int_{A_{m_{0}}}\left(\left\|F_{n}(t)\right\|\left\|f_{n}(t)\right\|-F_{n}(t)\left(f_{n}(t)\right)\right) \mathrm{d} \mu(t) \leq \varepsilon
\end{gathered}
$$

hold.

It follows that for every $n \geq N$ we have

$$
\begin{aligned}
& \left|\int_{A_{m_{0}}}\left\|F_{n}(t)\right\|\|f(t)\| \mathrm{d} \mu(t)-1\right| \\
& \quad \leq\|\| f(\cdot)\left\|\chi_{S \backslash A_{m_{0}}}\right\|_{E}+\left|\int_{S}\left\|F_{n}(t)\right\|\|f(t)\| \mathrm{d} \mu(t)-1\right| \leq \delta,
\end{aligned}
$$

and hence by the choice of $\delta$,

$$
\int_{A_{m_{0}}}\left\|F_{n}(t)\right\|\left\|f_{n}(t)\right\| \mathrm{d} \mu(t) \geq 1-\varepsilon .
$$

Consequently, for every $n \geq N$ we have

$$
\begin{aligned}
& \int_{S}\left(\left\|F_{n}(t)\right\|\left\|f_{n}(t)\right\|-F_{n}(t)\left(f_{n}(t)\right)\right) \mathrm{d} \mu(t) \\
& \quad \leq \varepsilon+\int_{S \backslash A_{m_{0}}}\left(\left\|F_{n}(t)\right\|\left\|f_{n}(t)\right\|-F_{n}(t)\left(f_{n}(t)\right)\right) \mathrm{d} \mu(t) \\
& \quad \leq \varepsilon+2 \int_{S \backslash A_{m_{0}}}\left\|F_{n}(t)\right\|\left\|f_{n}(t)\right\| \mathrm{d} \mu(t) \\
& \quad \leq \varepsilon+2(1-(1-\varepsilon))=3 \varepsilon .
\end{aligned}
$$

Thus we have shown

$$
\lim _{n \rightarrow \infty} \int_{S}\left(\left\|F_{n}(t)\right\|\left\|f_{n}(t)\right\|-F_{n}(t)\left(f_{n}(t)\right)\right) \mathrm{d} \mu(t)=0 .
$$

Together with (168) it follows $l_{n}\left(f_{n}\right) \rightarrow 1$, as desired.
Now let us treat the case of uacs spaces. In analogy to [4, Definition 3.15] we say that an order continuous Köthe function space $E$ has property $\left(u^{+}\right)$if for every $\varepsilon>0$ there is some $\delta>0$ such that for all $f, g \in S_{E}$ and every $h \in S_{E^{\prime}}$ we have

$$
\begin{gathered}
\|f+g\|_{E} \geq 2(1-\delta), \\
\int_{S} f h \mathrm{~d} \mu=1 \Longrightarrow \int_{S}|h||f-g| \mathrm{d} \mu \leq \varepsilon .
\end{gathered}
$$

This property certainly implies that $E$ is uacs, while every UR space has property $\left(u^{+}\right)$, but the author does not know whether these implications are strict.

The following theorem holds. Its proof is completely analogous to the one of [4, Theorem 3.16] (which is a modification of the proof of [20, Theorem 3]), but we will explicitly give it here, for the readers convenience.

Theorem 20. If $E$ is an order continuous Köthe function space with the property $\left(u^{+}\right)$(in particular, if $E$ is $U R$ ) and $X$ is a uacs Banach space, then $E(X)$ is also uacs.

Proof. Let $0<\varepsilon \leq 2$ be arbitrary. Since $E$ is in particular uacs there is a number $\eta>0$ such that for all functions $a, b \in B_{E}$ and every functional $l \in B_{E^{*}}$ with $l(a)=1$ one has

$$
l(b)<1-\frac{\varepsilon}{4} \delta_{\text {uacs }}^{X}\left(\frac{\varepsilon}{2}\right) \Longrightarrow\|a+b\|_{E} \leq 2(1-\eta) .
$$

Now let $f, g \in S_{E(X)}$ such that $\|f(t)\|=\|g(t)\|$ a.e., and let $L \in E(X)^{*}$ such that $L(f)=1$ and $L(g)<1-\varepsilon$. We claim that $\|f+g\|_{E(X)} \leq 2(1-\eta)$.

Let $L$ be represented by $[F] \in E^{\prime}\left(X^{*}, w^{*}\right)$, and put $\beta=$ $\|g(\cdot)\|, v=\|F(\cdot)\|$. Define $\gamma$ by $\gamma(t)=\nu(t) \beta(t)-F(t)(g(t))$. Note that $\gamma$ is measurable and

$$
0 \leq \gamma(t) \leq 2 \nu(t) \beta(t) \quad \forall t \in S .
$$

As before we can deduce from $L(f)=1$ that

$$
\int_{S}\|F(t)\|\|f(t)\| \mathrm{d} \mu(t)=1
$$

and $F(t)(f(t))=\|F(t)\|\|f(t)\|$ a.e., hence

$$
F(t)(f(t))=v(t) \beta(t) \quad \text { a.e. }
$$

Next we define

$$
\alpha(t)= \begin{cases}\frac{1}{2} \delta_{\text {uacs }}^{X}\left(\frac{\gamma(t)}{\nu(t) \beta(t)}\right) & \text { if } 0<\gamma(t)<\nu(t) \beta(t), \\ 0 & \text { if } \gamma(t)=0, \\ \frac{1}{2} \delta_{\text {uacs }}^{X}(1) & \text { otherwise. }\end{cases}
$$

Note that since $\delta_{\text {uacs }}^{X}$ is continuous on $(0,1)$ (see [21, Lemma 3.10] or [4, Lemma 2.11]), the function $\alpha$ is measurable. Using (188) it is easy to see that

$$
\|f(t)+g(t)\| \leq 2(1-\alpha(t)) \beta(t) \quad \text { a.e. }
$$


By (186) and (187) we have $\int_{S} \gamma(t) \mathrm{d} \mu(t) \leq 2$. Furthermore, we also have

$$
\begin{aligned}
\varepsilon & <1-L(g)=L(f-g)=\int_{S} F(t)(f(t)-g(t)) \mathrm{d} \mu(t) \\
& \leq \int_{S} \gamma(t) \mathrm{d} \mu(t) ;
\end{aligned}
$$

thus

$$
\varepsilon<\int_{S} \gamma(t) \mathrm{d} \mu(t) \leq 2
$$

Now put $A=\{t \in S: 2 \gamma(t)>\varepsilon \nu(t) \beta(t)\}$ and $B=S \backslash A$. We then have (because of (187))

$$
\begin{aligned}
\int_{B} \gamma(t) \mathrm{d} \mu(t) & \leq \frac{\varepsilon}{2} \int_{B} \nu(t) \beta(t) \mathrm{d} \mu(t) \\
& \leq \frac{\varepsilon}{2} \int_{S} \nu(t) \beta(t) \mathrm{d} \mu(t)=\frac{\varepsilon}{2}
\end{aligned}
$$

Together with (192) it follows that

$$
\int_{A} \gamma(t) \mathrm{d} \mu(t)>\varepsilon-\frac{\varepsilon}{2}=\frac{\varepsilon}{2} .
$$

Taking into account (186) we get

$$
\int_{A} \nu(t) \beta(t) \mathrm{d} \mu(t)>\frac{\varepsilon}{4} .
$$

Next we define $h=\beta \chi_{B}$ and $h^{\prime}=\beta \chi_{A}$ as well as $h^{\prime \prime}=(1-$ $\left.\delta_{\text {uacs }}^{X}(\varepsilon / 2)\right) h^{\prime}$. Then $\left\|h+h^{\prime \prime}\right\|_{E} \leq\left\|h+h^{\prime}\right\|_{E}=\|\beta\|_{E}=1$. Let $l$ be the functional on $E$ represented by $v=\|F(\cdot)\|$. We have $l\left(h+h^{\prime}\right)=l(\beta)=1$ (by (187)) and further, by (195),

$$
\begin{aligned}
l\left(h+h^{\prime \prime}\right) & =1-\delta_{\text {uacs }}^{X}\left(\frac{\varepsilon}{2}\right) l\left(h^{\prime}\right) \\
& =1-\int_{A} \nu(t) \beta(t) \mathrm{d} \mu(t)<1-\frac{\varepsilon}{4} \delta_{\text {uacs }}^{X}\left(\frac{\varepsilon}{2}\right) .
\end{aligned}
$$

So by our choice of $\eta$ we get $\left\|2 h+h^{\prime}+h^{\prime \prime}\right\|_{E} \leq 2(1-\eta)$; that is,

$$
\left\|h+\left(1-\frac{1}{2} \delta_{\text {uacs }}^{X}\left(\frac{\varepsilon}{2}\right)\right) h^{\prime}\right\|_{E} \leq 1-\eta .
$$

By monotonicity of $\delta_{\text {uacs }}^{X}$ we have

$$
\alpha(t) \geq \frac{1}{2} \delta_{\text {uacs }}^{X}\left(\frac{\varepsilon}{2}\right) \quad \forall t \in A
$$

Using (190), (198), and (197) we obtain

$$
\begin{aligned}
\|f+g\|_{E(X)} & =\|\| f(\cdot)+g(\cdot)\|\|_{E} \leq 2\|(1-\alpha) \beta\|_{E} \\
& \leq 2\left\|\left(1-2^{-1} \delta_{\text {uacs }}^{X}\left(\frac{\varepsilon}{2}\right)\right) h^{\prime}+h\right\|_{E} \leq 2(1-\eta) .
\end{aligned}
$$

The first step of the proof is completed. Next we wish to remove the restriction $\|f(\cdot)\|=\|g(\cdot)\|$ a.e. So let again $0<$ $\varepsilon \leq 2$ be arbitrary, and choose $\eta$ as above but corresponding to the value $\varepsilon / 2$. Take $0<\omega<2 \eta / 3$.

Since $E$ is uacs, we may find $\tau>0$ such that for all $a, b \in$ $B_{E}$ and every $l \in B_{E^{*}}$ we have

$$
l(a) \geq 1-\tau, \quad\|a+b\|_{E} \geq 2(1-\tau) \Longrightarrow l(b) \geq 1-\omega .
$$

Next we fix $0<\rho<\min \{\varepsilon / 2,2 \tau, \omega\}$ and find a number $\tilde{\tau}$ to the value $\rho$ according to the definition of the property $\left(u^{+}\right)$of $E$. Finally, let $0<\xi<\min \{\tau, \tilde{\tau}\}$.

Let $f, g \in S_{E(X)}$ be arbitrary and $L \in S_{E(X)^{*}}$ (as usually represented by $F$ ) such that $L(f)=1$ and $\|f+g\|_{E(X)} \geq 2(1-$ $\xi)$. We are going to prove that $L(g)>1-\varepsilon$, thus showing that $E(X)$ is uacs.

To this end, we define $z: S \rightarrow X$ by

$$
z(t)= \begin{cases}\frac{\|f(t)\|}{\|g(t)\|} g(t) & \text { if } g(t) \neq 0, \\ f(t) & \text { if } g(t)=0 .\end{cases}
$$

Then $z$ is Bochner-measurable, and $\|z(t)\|=\|f(t)\|$ for all $t \in S$ (hence $z \in E(X)$ ). Furthermore,

$$
\|z(t)-g(t)\|=|\|f(t)\|-\|g(t)\|| \quad \forall t \in S .
$$

As before we have

$$
\int_{S}\|F(t)\|\|f(t)\| \mathrm{d} \mu(t)=1 .
$$

Also,

$$
2(1-\tilde{\tau}) \leq 2(1-\xi) \leq\|f+g\|_{E(X)} \leq\|\| f(\cdot)\|+\| g(\cdot)\|\|_{E},
$$

so the choice of $\widetilde{\tau}$ together with (202) implies

$$
\int_{S}\|F(t)\|\|z(t)-g(t)\| \mathrm{d} \mu(t) \leq \rho .
$$

Next we observe that

$$
\begin{aligned}
& \|\| f(\cdot)\|+\| g(\cdot)\|+\| f(\cdot)+g(\cdot)\|\|_{E} \\
& \quad \geq 2\|f+g\|_{E(X)} \geq 4(1-\xi) \geq 4(1-\tau),
\end{aligned}
$$

and (because of (203) and (205))

$$
\begin{aligned}
\int_{S}\|F(t)\|(\|f(t)\|+\|g(t)\|) \mathrm{d} \mu(t) \\
=1+\int_{S}\|F(t)\|\|g(t)\| \mathrm{d} \mu(t) \\
\geq 1+\int_{S}\|F(t)\|\|f(t)\| \mathrm{d} \mu(t) \\
\quad-\int_{S}\|F(t)\| \mid\|f(t)\|-\|g(t)\| \mathrm{d} \mu(t) \\
=2-\int_{S}\|F(t)\| \mid\|f(t)\|-\|g(t)\| \mathrm{d} \mu(t) \\
\geq 2-\rho \geq 2(1-\tau) .
\end{aligned}
$$


So (200) implies

$$
\int_{S}\|F(t)\|\|f(t)+g(t)\| \mathrm{d} \mu(t) \geq 2(1-\omega) .
$$

Using (205) and (208) we can conclude

$$
\begin{aligned}
\|f+z\|_{E(X)} \geq & \int_{S}\|F(t)\|\|f(t)+z(t)\| \mathrm{d} \mu(t) \\
\geq & \int_{S}\|F(t)\|\|f(t)+g(t)\| \mathrm{d} \mu(t) \\
& -\int_{S}\|F(t)\|\|g(t)-z(t)\| \mathrm{d} \mu(t) \\
\geq & 2(1-\omega)-\rho>2(1-\eta) .
\end{aligned}
$$

By the choice of $\eta$ this implies $L(z) \geq 1-\varepsilon / 2$. But by (205) we also have $|L(g)-L(z)| \leq \rho$; hence $L(g) \geq L(z)-\rho \geq$ $1-\varepsilon / 2-\rho>1-\varepsilon$.

The above theorem admits the following corollary.

Corollary 21. If $E$ is a US Köthe function space and $X$ is a uacs Banach space, and hence also $E(X)$ is uacs.

Proof. Since uacs is a self-dual property (cf. [4, Corollary 2.13]) $X^{*}$ is also uacs, and since $E$ is US, we have that $E^{*}=E^{\prime}$ is UR (cf. [2, Theorem 9.10]). So by the previous theorem $E^{\prime}\left(X^{*}\right)$ is uacs. But as a uacs space, $X^{*}$ is reflexive, and hence it has the Radon-Nikodým property. It follows from the general theory in [13] that in this case $E(X)^{*}$ is isometrically isomorphic to $E^{\prime}\left(X^{*}\right)$, so $E(X)^{*}$ and hence also $E(X)$ is uacs.

Finally, we consider some midpoint version of luacs and sluacs spaces. Let us first recall the following well-known notions: a Banach space $X$ is said to be midpoint locally uniformly rotund (MLUR) if for any two sequences $\left(x_{n}\right)_{n \in \mathbb{N}}$ and $\left(y_{n}\right)_{n \in \mathbb{N}}$ in $S_{X}$ and every $x \in S_{X}$ we have

$$
\left\|x-\frac{x_{n}+y_{n}}{2}\right\| \longrightarrow 0 \Longrightarrow\left\|x_{n}-y_{n}\right\| \longrightarrow 0 .
$$

$X$ is called weakly midpoint locally uniformly rotund (WMLUR) if it satisfies the above condition with $\left\|x_{n}-y_{n}\right\| \rightarrow 0$ replaced by $x_{n}-y_{n} \stackrel{\sigma}{\rightarrow} 0$, where the symbol $\stackrel{\sigma}{\rightarrow}$ denotes the convergence in the weak topology of $X$. The notion of MLUR spaces was originally introduced by Anderson in [22].

In [4] the author introduced the following analogous definitions.

Definition 22. Let $X$ be a Banach space.

(i) The space $X$ is said to be midpoint locally uniformly alternatively convex or smooth (mluacs) if for any two sequences $\left(x_{n}\right)_{n \in \mathbb{N}}$ and $\left(y_{n}\right)_{n \in \mathbb{N}}$ in $S_{X}$, every $x \in S_{X}$, and every $x^{*} \in S_{X^{*}}$, one has

$$
\left\|x-\frac{x_{n}+y_{n}}{2}\right\| \longrightarrow 0, \quad x^{*}\left(x_{n}\right) \longrightarrow 1 \Longrightarrow x^{*}\left(y_{n}\right) \longrightarrow 1 .
$$

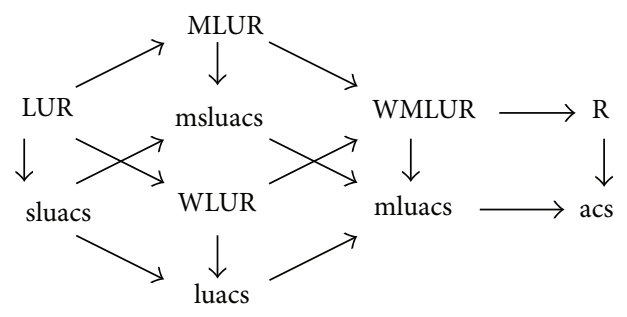

FIGURE 4

(ii) The space $X$ is called midpoint strongly locally uniformly alternatively convex or smooth (msluacs) if for any two sequences $\left(x_{n}\right)_{n \in \mathbb{N}}$ and $\left(y_{n}\right)_{n \in \mathbb{N}}$ in $S_{X}$, every $x \in S_{X}$ and every sequence $\left(x_{n}^{*}\right)_{n \in \mathbb{N}}$ in $S_{X^{*}}$, one has

$$
\left\|x-\frac{x_{n}+y_{n}}{2}\right\| \longrightarrow 0, \quad x_{n}^{*}\left(x_{n}\right) \longrightarrow 1 \Longrightarrow x_{n}^{*}\left(y_{n}\right) \longrightarrow 1 .
$$

Figure 4 summarises the obvious implications. No other implications are true in general (see the examples in [4]).

Concerning the properties msluacs and mluacs for Köthe-Bochner spaces we have the following result.

Theorem 23. Let $E$ be an MLUR Köthe function space over a complete $\sigma$-finite measure space and $X$ a Banach space. If $X$ is mluacs, then so is $E(X)$. If $X$ is msluacs and in addition $E^{*}$ has the Kadets-Klee* property and $B_{E^{*}}$ is weak ${ }^{*}$-sequentially compact, then $E(X)$ is also msluacs.

Proof. Let us first recall that $\ell^{\infty}$ has no equivalent MLUR norm (cf. [11, Theorem 2.1.5]), and so by [11, Propositions 3.1.4 and 3.1.5] (and since every Köthe function space is $\sigma$ order complete), $E$ must be order continuous.

Now let us assume that $X$ is msluacs and $E^{*}$ has the Kadets-Klee ${ }^{*}$ property and weak ${ }^{*}$-sequentially compact unit ball. To show that $E(X)$ is msluacs we will proceed in an analogous way to the proof of [4, Proposition 4.7], which in turn uses techniques from the proof of [23, Proposition 4].

So let us take two sequences $\left(f_{n}\right)_{n \in \mathbb{N}},\left(g_{n}\right)_{n \in \mathbb{N}}$ in $S_{E(X)}$ and $f \in S_{E(X)}$ such that $\left\|f_{n}+g_{n}-2 f\right\|_{E(X)} \rightarrow 0$. Also, take a sequence $\left(l_{n}\right)_{n \in \mathbb{N}}$ of norm-one functionals on $E(X)$ such that $l_{n}\left(f_{n}\right) \rightarrow 1$. As usual, $l_{n}$ will be represented by $\left[F_{n}\right] \in$ $E^{\prime}\left(X^{*}, w^{*}\right)$, and we conclude

$$
\lim _{n \rightarrow \infty} \int_{S}\left\|F_{n}(t)\right\|\left\|f_{n}(t)\right\| \mathrm{d} \mu(t)=1
$$

and, after passing to an appropriate subsequence,

$$
\lim _{n \rightarrow \infty}\left(\left\|F_{n}(t)\right\|\left\|f_{n}(t)\right\|-F_{n}(t)\left(f_{n}(t)\right)\right)=0 \quad \text { a.e. }
$$

We also have

$$
\begin{aligned}
\|2\| f(\cdot)\|-\| f_{n}(\cdot)+g_{n}(\cdot)\|\|_{E} \\
\quad=\left\|\left|2\|f(\cdot)\|-\left\|f_{n}(\cdot)+g_{n}(\cdot)\right\|\right|\right\|_{E} \\
\quad \leq\|\| 2 f(\cdot)-f_{n}(\cdot)-g_{n}(\cdot)\|\|_{E}=\left\|2 f-f_{n}-g_{n}\right\|_{E(X)} ;
\end{aligned}
$$


hence

$$
\|2\| f(\cdot)\|-\| f_{n}(\cdot)+g_{n}(\cdot)\|\|_{E} \longrightarrow 0
$$

As before we can also show

$$
\|\| f_{n}(\cdot)\|+\| g_{n}(\cdot)\|\|_{E} \longrightarrow 2 .
$$

Also, because of $\left\|f_{n}+g_{n}-2 f\right\|_{E(X)} \rightarrow 0$, we may pass to a further subsequence such that

$$
\lim _{n \rightarrow \infty}\left\|f_{n}(t)+g_{n}(t)-2 f(t)\right\|=0 \quad \text { a.e. }
$$

Let us define for every $n \in \mathbb{N}$

$$
\begin{gathered}
a_{n}(t):=2\|f(t)\|-\frac{1}{2}\left(\left\|f_{n}(t)\right\|+\left\|g_{n}(t)\right\|\right), \\
b_{n}(t):=\|f(t)\|-\frac{1}{2}\left\|f_{n}(t)+g_{n}(t)\right\| .
\end{gathered}
$$

Note that

$$
\|f(t)\| \leq b_{n}(t)+\frac{1}{2}\left(\left\|f_{n}(t)\right\|+\left\|g_{n}(t)\right\|\right) .
$$

So if $a_{n}(t) \geq 0$, then

$$
\begin{aligned}
\left|a_{n}(t)\right| & =2\|f(t)\|-\frac{1}{2}\left(\left\|f_{n}(t)\right\|+\left\|g_{n}(t)\right\|\right) \\
& \leq 2\left|b_{n}(t)\right|+\frac{1}{2}\left(\left\|f_{n}(t)\right\|+\left\|g_{n}(t)\right\|\right) .
\end{aligned}
$$

If $a_{n}(t)<0$, then

$$
\begin{aligned}
\left|a_{n}(t)\right| & =\frac{1}{2}\left(\left\|f_{n}(t)\right\|+\left\|g_{n}(t)\right\|\right)-2\|f(t)\| \\
& \leq 2\left|b_{n}(t)\right|+\frac{1}{2}\left(\left\|f_{n}(t)\right\|+\left\|g_{n}(t)\right\|\right) .
\end{aligned}
$$

So we always have

$$
\left|a_{n}(t)\right| \leq 2\left|b_{n}(t)\right|+\frac{1}{2}\left(\left\|f_{n}(t)\right\|+\left\|g_{n}(t)\right\|\right) .
$$

It follows that

$$
\begin{aligned}
& \frac{1}{2}\|\| f_{n}(\cdot)\|+\| g_{n}(\cdot)\|\|_{E}+2\left\|b_{n}\right\|_{E} \\
& \geq\left\|2\left|b_{n}\right|+\frac{1}{2}\left(\left\|f_{n}(\cdot)\right\|+\left\|g_{n}(\cdot)\right\|\right)\right\|_{E} \\
& \geq\left\|a_{n}\right\|_{E} \geq 2-\frac{1}{2}\|\| f_{n}(\cdot)\|+\| g_{n}(\cdot)\|\|_{E},
\end{aligned}
$$

and we can conclude with (216) and (217) that $\left\|a_{n}\right\|_{E} \rightarrow 1$.

Using this together with (216), $\left\|f_{n}(\cdot)\right\|+\left\|g_{n}(\cdot)\right\|+2 a_{n}=$ $4\|f(\cdot)\|$, and the fact that $E$ is MLUR we get

$$
\lim _{n \rightarrow \infty}\|2\| f(\cdot)\|-\| f_{n}(\cdot)\|-\| g_{n}(\cdot)\|\|_{E}=0 .
$$

Again, since $E$ is MLUR, this implies

$$
\lim _{n \rightarrow \infty}\|\| f_{n}(\cdot)\|-\| g_{n}(\cdot)\|\|_{E}=0 .
$$

Because of (225) and (226), we can pass to a further subsequence such that

$$
\lim _{n \rightarrow \infty}\left\|f_{n}(t)\right\|=\|f(t)\|=\lim _{n \rightarrow \infty}\left\|g_{n}(t)\right\| \quad \text { a.e. }
$$

Since $B_{E^{*}}$ is weak ${ }^{*}$-sequentially compact we may also asssume that $\left(\left\|F_{n}(\cdot)\right\|\right)_{n \in \mathbb{N}}$ weak $^{*}$-converges to some $g \in B_{E^{\prime}}$.

Equations (225) and (226) imply \|\|$f_{n}(\cdot)\|-\| f(\cdot)\|\|_{E} \rightarrow$ 0 . Together with (213) this gives us

$$
\lim _{n \rightarrow \infty} \int_{S}\left\|F_{n}(t)\right\|\|f(t)\| \mathrm{d} \mu(t)=1
$$

hence we also have

$$
\int_{S}\|g(t)\|\|f(t)\| \mathrm{d} \mu(t)=1 ;
$$

thus $\|g\|_{E^{\prime}}=1$. Since $E^{*}$ has the Kadets-Klee* property, it follows that \|\|$F_{n}(\cdot)\|-g\|_{E^{\prime}} \rightarrow 0$, so if we pass again to a subsequence, we may assume

$$
\lim _{n \rightarrow \infty}\left\|F_{n}(t)\right\|=g(t) \quad \text { a.e. }
$$

Now if we combine (214), (216), (227), and (230) we obtain

$$
\lim _{n \rightarrow \infty}\left(\left\|F_{n}(t)\right\|\|f(t)\|-F_{n}(t)(f(t))\right)=0 \quad \text { a.e., }
$$

since $X$ is msluacs.

Using our usual argument via equi-integrability and Vitali's lemma this leads to

$$
\lim _{n \rightarrow \infty} \int_{A}\left(\left\|F_{n}(t)\right\|\|f(t)\|-F_{n}(t)(f(t))\right) \mathrm{d} \mu(t)=0
$$

for every $A \in \mathscr{A}$ with $\mu(A)<\infty$.

By the order continuity of $E$ we can derive from this

$$
\lim _{n \rightarrow \infty} \int_{S}\left(\left\|F_{n}(t)\right\|\|f(t)\|-F_{n}(t)(f(t))\right) \mathrm{d} \mu(t)=0
$$

also in the $\sigma$-finite case (cf. the proof of Theorem 18).

Combining (233) and (228) gives us $l_{n}(f) \rightarrow 1$, and we are done.

The statement about mluacs spaces can be proved similarly.

We remark that the results proved in this section especially apply to $L^{p}$ spaces for $1<p<\infty$ (as we said before, for the properties acs/luacs/uacs this was already proved by Sirotkin in [5]).

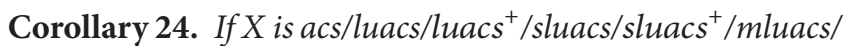
msluacs/wuacs/uacs, then for any complete, $\sigma$-finite measure space $(S, \mathscr{A}, \mu)$ and any $1<p<\infty$ the Lebesgue-Bochner space $L^{p}(\mu)(X)$ has the same property.

In the last section we will establish some further connections between the various properties that we considered in this paper. 


\section{Miscellaneous}

In [24] Lovaglia called a Banach space $X$ weakly locally uniformly rotund if for every sequence $\left(x_{n}\right)_{n \in \mathbb{N}}$ in $S_{X}$, every $x \in S_{X}$ and each $x^{*} \in S_{X^{*}}$, the implication

$$
\left\|x_{n}+x\right\| \longrightarrow 2, \quad x^{*}(x)=1 \Longrightarrow x^{*}\left(x_{n}\right) \longrightarrow 1
$$

holds. Since this notion of weak local uniform rotundity is strictly weaker than the notion of WLUR spaces that is nowadays commonly used, we will call such spaces WLUR in the sense of Lovaglia. (A dual Banach space will be called WLUR ${ }^{*}$ in the sense of Lovaglia, if it fulfils Lovaglia's definition for all evaluation functionals.) By definition, a Banach space is luacs ${ }^{+}$if and only if it is luacs and WLUR in the sense of Lovaglia. Also, the following is valid.

Proposition 25. A Banach space $X$ is luacs ${ }^{+}$if and only if $X$ is WLUR in the sense of Lovaglia and for all $x^{*}, y^{*} \in S_{X^{*}}$ with $\left\|x^{*}+y^{*}\right\|=2$ and every $x \in S_{X}$ with $x^{*}(x)=1$ one also has $y^{*}(x)=1$.

Proof. The necessity is clear because of [4, Proposition 2.16 (i)]. For the sufficiency we only have to prove that $X$ is luacs, so let us take a sequence $\left(x_{n}\right)_{n \in \mathbb{N}}$ in $S_{X}$ and $x \in S_{X}$ such that $\left\|x_{n}+x\right\| \rightarrow 2$ as well as $x^{*} \in S_{X^{*}}$ with $x^{*}\left(x_{n}\right) \rightarrow 1$. Since $B_{X^{* *}}$ is weak ${ }^{*}$-compact, we can find $x^{* *} \in B_{X^{* *}}$ and a subnet $\left(x_{\varphi(i)}\right)_{i \in I}$ which is weak ${ }^{*}$-convergent to $x^{* *}$. It follows that $x^{* *}\left(x^{*}\right)=1=\left\|x^{* *}\right\|$.

Now fix a sequence $\left(y_{n}^{*}\right)_{n \in \mathbb{N}}$ in $S_{X^{*}}$ such that $y_{n}^{*}\left(x_{n}+x\right) \rightarrow$ 2 . Then $y_{n}^{*}\left(x_{n}\right) \rightarrow 1$ and $y_{n}^{*}(x) \rightarrow 1$. There are $y^{*} \in B_{X^{*}}$ and a subnet $\left(y_{\psi(j)}^{*}\right)_{j \in J}$ which is weak ${ }^{*}$-convergent to $y^{*}$. It follows that $y^{*}(x)=1=\left\|y^{*}\right\|$. Since $X$ is WLUR in the sense of Lovaglia, we conclude $y^{*}\left(x_{n}\right) \rightarrow 1$. It follows that $x^{* *}\left(y^{*}\right)=$ $1=x^{* *}\left(x^{*}\right)$; hence $\left\|x^{*}+y^{*}\right\|=2$.

Because of $y^{*}(x)=1$, our assumption implies $x^{*}(x)=1$ and we are done. details).

The following assertion is also easy to prove (we omit the

Proposition 26. If $X$ is a Banach space which is WLUR in the sense of Lovaglia, and such that $X^{*}$ is WLUR ${ }^{*}$ in the sense of Lovaglia then $X$ is sluacs.

Under additional assumptions on the space $X$ it is possible to prove some more results.

\section{Proposition 27. Let $X$ be a reflexive Banach space.}

(i) If $X$ is WLUR in the sense of Lovaglia, then $X$ is luacs ${ }^{+}$.

(ii) If $X$ is sluacs and luacs ${ }^{+}$, then $X$ is wuacs.

(iii) If $X$ is wuacs and $R$, then $X$ is WLUR.

Proof. (i) follows directly from the Proposition 25 and [4, Proposition 2.15]. Of the remaining assertions we will only prove (iii) explicitly.

Let $\left(x_{n}\right)_{n \in \mathbb{N}}$ be a sequence in $S_{X}$ and $x \in S_{X}$ such that $\left\|x_{n}+x\right\| \rightarrow 2$. We can find a sequence $\left(x_{n}^{*}\right)_{n \in \mathbb{N}}$ in $S_{X^{*}}$ such that $x_{n}^{*}\left(x_{n}+x\right) \rightarrow 2$, and hence $x_{n}^{*}\left(x_{n}\right) \rightarrow 1$ and $x_{n}^{*}(x) \rightarrow 1$.
Since $X$ is reflexive, we may assume that $\left(x_{n}^{*}\right)_{n \in \mathbb{N}}$ is weak $^{*}$-convergent to some $y^{*} \in B_{X^{*}}$ and $\left(x_{n}\right)_{n \in \mathbb{N}}$ is weakly convergent to some $y \in B_{X}$. It follows that $y^{*}(x)=1$ and hence $\left\|x_{n}^{*}+y^{*}\right\| \rightarrow 2$.

Since $X$ is wuacs, the dual space $X^{*}$ is sluacs (cf. [4, Proposition 2.16]), and thus (because of $x_{n}^{*}\left(x_{n}\right) \rightarrow 1$ ) we can conclude that $y^{*}\left(x_{n}\right) \rightarrow 1$, whence $y^{*}(y)=1=y^{*}(x)$, which implies $\|x+y\|=2$, which by the rotundity of $X$ implies $x=y$.

Proposition 28. Let $X$ be a reflexive Banach space with the Kadets-Klee property.

(i) If $X$ is acs then $X$, is luacs.

(ii) If $X$ is WLUR in the sense of Lovaglia, then $X$ is wuacs and sluacs ${ }^{+}$.

(iii) If $X$ is WLUR in the sense of Lovaglia and $R$, then $X$ is wuacs and $L U R$.

Proof. (i) Let $\left(x_{n}\right)_{n \in \mathbb{N}}, x$, and $y$ be as in the proof of (iii) of the previous proposition and let $x^{*} \in S_{X^{*}}$ with $x^{*}\left(x_{n}\right) \rightarrow 1$. Then $x^{*}(y)=1$ and hence $\|y\|=1$. Since $X$ has the KadetsKlee property, it follows that $\left\|x_{n}-y\right\| \rightarrow 0$ and thus $\|x+y\|=$ 2. Because $X$ is acs, we obtain $x^{*}(x)=1$, as desired.

(ii) We first show that $X$ is wuacs. Take two sequences $\left(x_{n}\right)_{n \in \mathbb{N}}$ and $\left(y_{n}\right)_{n \in \mathbb{N}}$ in $S_{X}$ such that $\left\|x_{n}+y_{n}\right\| \rightarrow 2$ and a functional $x^{*} \in S_{X^{*}}$ with $x^{*}\left(x_{n}\right) \rightarrow 1$. By the reflexivity of $X$ we may assume that $\left(x_{n}\right)_{n \in \mathbb{N}}$ is weakly convergent to some $x \in B_{X}$. Then $x^{*}(x)=1$; hence $\|x\|=1$.

But $X$ has the Kadets-Klee property, so this implies $\| x_{n}-$ $x \| \rightarrow 0$.

Now fix a sequence $\left(y_{n}^{*}\right)_{n \in \mathbb{N}}$ in $S_{X^{*}}$ such that $y_{n}^{*}\left(x_{n}\right) \rightarrow$ 1 , and $y_{n}^{*}\left(y_{n}\right) \rightarrow 1$. It follows that $y_{n}^{*}(x) \rightarrow 1$ and consequently $\left\|y_{n}+x\right\| \rightarrow 2$.

Since $x^{*}(x)=1$ and $X$ is WLUR in the sense of Lovaglia, we get $x^{*}\left(y_{n}\right) \rightarrow 1$, proving that $X$ is wuacs.

Now we will show that $X$ is sluacs. Take $\left(x_{n}\right)_{n \in \mathbb{N}}$ and $x$ in $S_{X}$ with $\left\|x_{n}+x\right\| \rightarrow 2$ and a sequence $\left(x_{n}^{*}\right)_{n \in \mathbb{N}}$ in $S_{X^{*}}$ such that $x_{n}^{*}\left(x_{n}\right) \rightarrow 1$. Also, fix a sequence $\left(y_{n}^{*}\right)_{n \in \mathbb{N}}$ in $S_{X^{*}}$ with $y_{n}^{*}\left(x_{n}\right) \rightarrow 1$ and $y_{n}^{*}(x) \rightarrow 1$.

We may assume that $\left(x_{n}\right)_{n \in \mathbb{N}}$ is weakly convergent to some $y \in B_{X}$ and $\left(y_{n}^{*}\right)_{n \in \mathbb{N}}$ is weak ${ }^{*}$-convergent to some $y^{*} \in B_{X^{*}}$. It follows that $y^{*}(x)=1$, and hence $\left\|y^{*}+y_{n}^{*}\right\| \rightarrow 2$.

Since $X$ is wuacs, $X^{*}$ is sluacs, and thus we get $y^{*}\left(x_{n}\right) \rightarrow$ 1. It follows that $y^{*}(y)=1$; hence $\|y\|=1$ and $\|x+y\|=2$. The Kadets-Klee property of $X$ gives us $\left\|x_{n}-y\right\| \rightarrow 0$.

Because of $x_{n}^{*}\left(x_{n}\right) \rightarrow 1$, we can now infer $x_{n}^{*}(y) \rightarrow 1$. Since $X$ is in particular acs, this implies $x_{n}^{*}(x) \rightarrow 1$ (cf. [4, Proposition 2.19]).

We will skip the last part of the proof, the reverse implication in the definition of sluacs ${ }^{+}$.

(iii) By (ii) $X$ is wuacs and sluacs ${ }^{+}$. Let us take a sequence $\left(x_{n}\right)_{n \in \mathbb{N}}$ in $S_{X}$ and an element $x \in S_{X}$ such that $\left\|x_{n}+x\right\| \rightarrow 2$. Fix a sequence $\left(x_{n}^{*}\right)_{n \in \mathbb{N}}$ in $S_{X^{*}}$ such that $x_{n}^{*}\left(x_{n}\right)=1$, for every $n \in \mathbb{N}$. Since $X$ is sluacs, it follows that $x_{n}^{*}(x) \rightarrow 1$.

Assume that $\left(x_{n}\right)_{n \in \mathbb{N}}$ is weakly convergent to $y \in B_{X}$ and that $\left(x_{n}^{*}\right)_{n \in \mathbb{N}}$ is weak ${ }^{*}$-convergent to $x^{*} \in B_{X^{*}}$. It follows that $x^{*}(x)=1$ and hence $x^{*} \in S_{X^{*}}$. Moreover, since $X$ is WLUR in the sense of Lovaglia, we get that $x^{*}\left(x_{n}\right) \rightarrow 1$. 
Since $\left(x_{n}\right)_{n \in \mathbb{N}}$ converges weakly to $y$ this implies $x^{*}(y)=$ 1 and hence $\|y\|=1$. Now the Kadets-Klee property of $X$ allows us to conclude $\left\|x_{n}-y\right\| \rightarrow 0$.

Because of $x^{*}(x)=x^{*}(y)=1$, we must have $\|x+y\|=2$ and thus the rotundity of $X$ implies $x=y$.

Proposition 29. Let $X$ be a Banach space such that $X^{*}$ has the Kadets-Klee ${ }^{*}$ property and $B_{X^{*}}$ is weak ${ }^{*}$-sequentially compact.

(i) If $X$ is $S$, then it is also WLUR in the sense of Lovaglia.

(ii) If $X^{*}$ is acs, then $X$ is luacs ${ }^{+}$, and for all sequences $\left(x_{n}\right)_{n \in \mathbb{N}}$ in $S_{X},\left(x_{n}^{*}\right)_{n \in \mathbb{N}}$ in $S_{X^{*}}$ and every $x \in S_{X}$ with $\left\|x_{n}+x\right\| \rightarrow 2$ and $x_{n}^{*}(x) \rightarrow 1$ one has $x_{n}^{*}\left(x_{n}\right) \rightarrow 1$.

(iii) If $X^{*}$ is WLUR* in the sense of Lovaglia, then $X$ is sluacs.

Proof. We will only prove (iii), so let $\left(x_{n}\right)_{n \in \mathbb{N}}$ and $x$ be in $S_{X}$ with $\left\|x_{n}+x\right\| \rightarrow 2$ and $\left(x_{n}^{*}\right)_{n \in \mathbb{N}}$ a sequence in $S_{X^{*}}$ such that $x_{n}^{*}\left(x_{n}\right) \rightarrow 1$. Let $\left(y_{n}^{*}\right)_{n \in \mathbb{N}}$ be a sequence in $S_{X^{*}}$ with $y_{n}^{*}\left(x_{n}\right) \rightarrow 1$ and $y_{n}^{*}(x) \rightarrow 1$.

By assumption, we may suppose that $\left(y_{n}^{*}\right)_{n \in \mathbb{N}}$ is weak ${ }^{*}$ convergent to some $y^{*} \in B_{X^{*}}$. Then $y^{*}(x)=1$; hence $y^{*} \in$ $S_{X^{*}}$. By the Kadets-Klee* property of $X^{*}$ we must have $\| y_{n}^{*}-$ $y^{*} \| \rightarrow 0$.

It follows that $y^{*}\left(x_{n}\right) \rightarrow 1$; hence $\left\|x_{n}^{*}+y^{*}\right\| \rightarrow 2$. Since $X^{*}$ is WLUR ${ }^{*}$ in the sense of Lovaglia, we obtain $x_{n}^{*}(x) \rightarrow$ 1 .

\section{References}

[1] M. A. Smith, "Some examples concerning rotundity in Banach spaces," Mathematische Annalen, vol. 233, no. 2, pp. 155-161, 1978.

[2] M. Fabian, P. Habala, P. Hájak et al., Functional Analysis and Infinite-Dimensional Geometry, CMS Books in Mathematics, Springer, New York, NY, USA, 2001.

[3] V. Kadets, R. Shvydkoy, G. Sirotkin, and D. Werner, "Banach spaces with the Daugavet property," Transactions of the American Mathematical Society, vol. 352, no. 2, pp. 855-873, 2000.

[4] J. D. Hardtke, "Absolute sums of Banach spaces and some geometric properties related to rotundity andsmoothness," http://arxiv.org/abs/1201.2300.

[5] G. G. Sirotkin, "New properties of Lebesgue-Bochner $L_{p}(\Omega$, $\Sigma, \mu ; X)$ spaces," Houston Journal of Mathematics, vol. 27, no. 4 , pp. 897-906, 2001.

[6] K. S. Lau, "Best approximation by closed sets in Banach spaces," Journal of Approximation Theory, vol. 23, no. 1, pp. 29-36, 1978.

[7] J. Gao, "Normal structure and modulus of $u$-convexity in Banach spaces," in Function Spaces, Differential Operators and Nonlinear Analysis, pp. 195-199, Prometheus, Prague, Czech Republic, 1996.

[8] S. Dhompongsa, A. Kaewkhao, and S. Saejung, "Uniform smoothness and $U$-convexity of $\psi$-direct sums," Journal of Nonlinear and Convex Analysis, vol. 6, no. 2, pp. 327-338, 2005.

[9] B. Beauzamy, Introduction to Banach Spaces and Their Geometry, North-Holland, Amsterdam, The Netherlands, 2nd edition, 1983.
[10] Y. A. Abramovich, C. D. Aliprantis, and O. Burkinshaw, "The Daugavet equation in uniformly convex Banach spaces," Journal of Functional Analysis, vol. 97, no. 1, pp. 215-230, 1991.

[11] P. K. Lin, Köthe-Bochner Function Spaces, Birkhäauser, Boston, Mass, USA, 2004.

[12] J. Lindenstrauss and L. Tzafriri, Classical Banach Spaces, vol. 2, Springer, Berlin, Germany, 1979.

[13] A. V. Bukhvalov, "On an analytic representation of operators with abstract norm," Soviet Mathematics Doklady, vol. 14, pp. 197-201, 1973.

[14] G. Emmanuele and A. Villani, "Lifting of rotundity properties from $E$ to $L^{p}(\mu, E)$," The Rocky Mountain Journal of Mathematics, vol. 17, no. 3, pp. 617-627, 1987.

[15] M. Fabian and S. Lajara, "Smooth renormings of the LebesgueBochner function space $L^{1}(\mu, X)$," Studia Mathematica, vol. 209, no. 3, pp. 247-265, 2012.

[16] A. Kamińska and B. Turett, "Rotundity in Köthe spaces of vector-valued functions," Canadian Journal of Mathematics, vol. 41, no. 4, pp. 659-675, 1989.

[17] M. A. Smith and B. Turett, "Rotundity in Lebesgue-Bochner function spaces," Transactions of the American Mathematical Society, vol. 257, no. 1, pp. 105-118, 1980.

[18] P. R. Halmos, Measure Theory, The University Series in Higher Mathematics, D. Van Nostrand, New York, NY, USA, 1950.

[19] N. L. Carothers, A Short Course on Banach Space Theory, vol. 64 of London Mathematical Society Student Texts, Cambridge University Press, Cambridge, UK, 2010.

[20] M. M. Day, “Uniform convexity. III," Bulletin of the American Mathematical Society, vol. 49, no. 10, pp. 745-750, 1943.

[21] S. Dhompongsa, A. Kaewkhao, and S. Tasena, "On a generalized James constant," Journal of Mathematical Analysis and Applications, vol. 285, no. 2, pp. 419-435, 2003.

[22] K. W. Anderson, Midpoint local uniform convexity, and other geometric properties of Banach spaces [Ph.D. dissertation], University of Illinois, 1960.

[23] P. N. Dowling and S. Saejung, "Extremal structure of the unit ball of direct sums of Banach spaces," Nonlinear Analysis: Theory, Methods \& Applications, vol. 68, no. 4, pp. 951-955, 2008.

[24] A. R. Lovaglia, "Locally uniformly convex Banach spaces," Transactions of the American Mathematical Society, vol. 78, no. 1, pp. 225-238, 1955. 


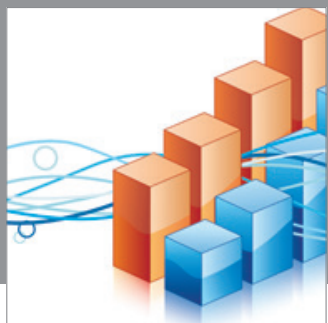

Advances in

Operations Research

mansans

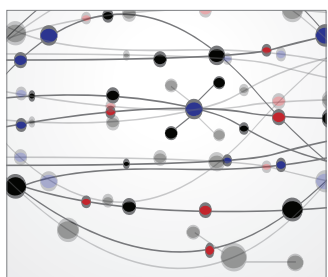

The Scientific World Journal
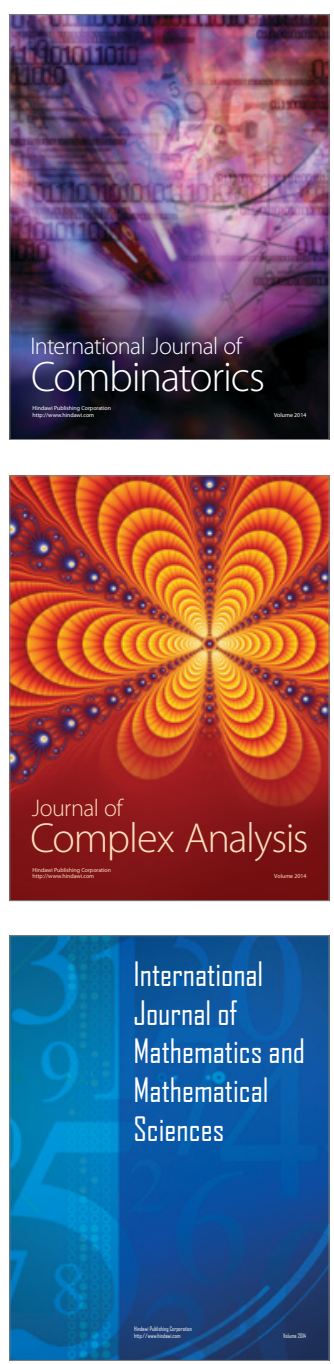
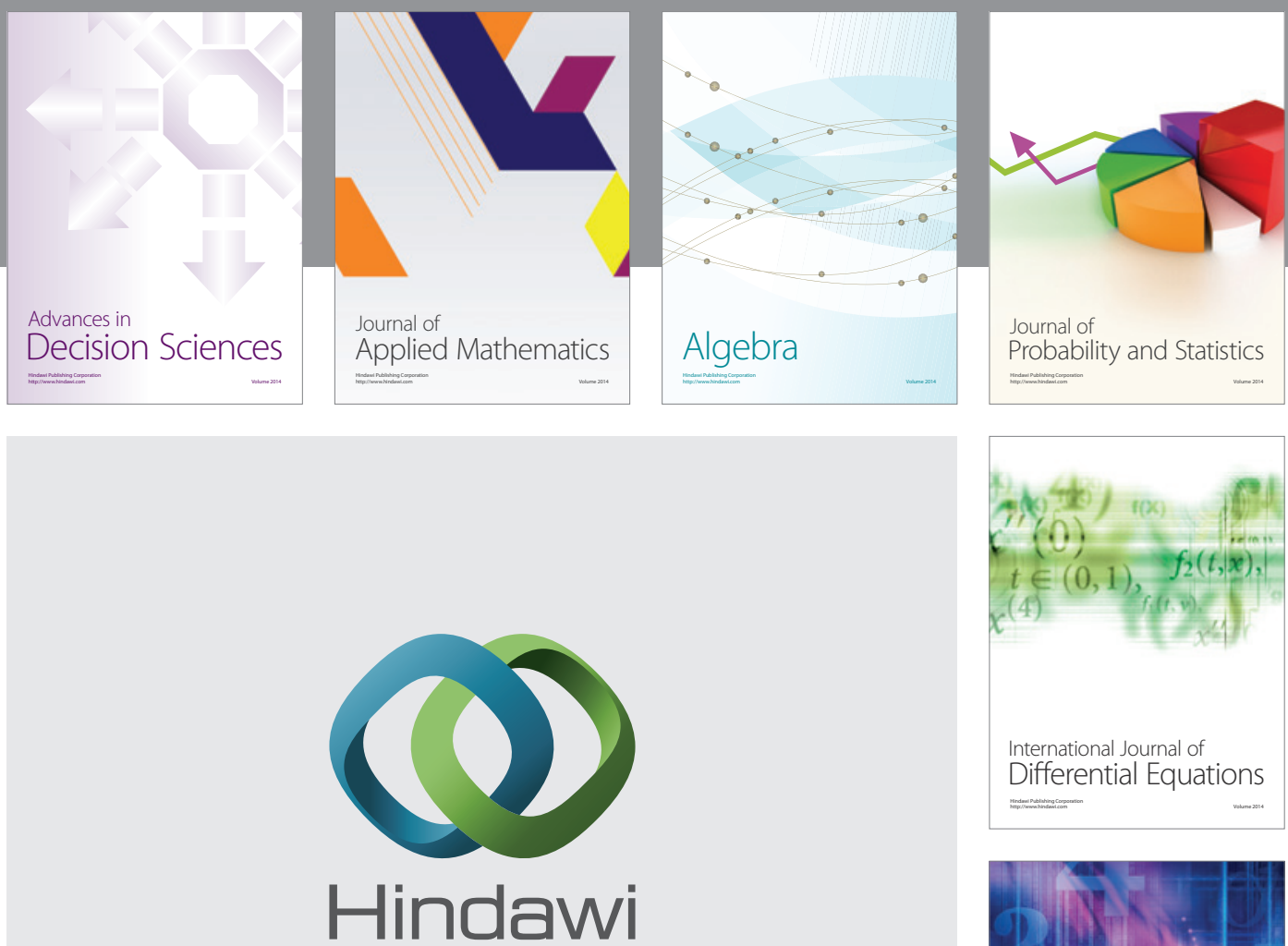

Submit your manuscripts at http://www.hindawi.com
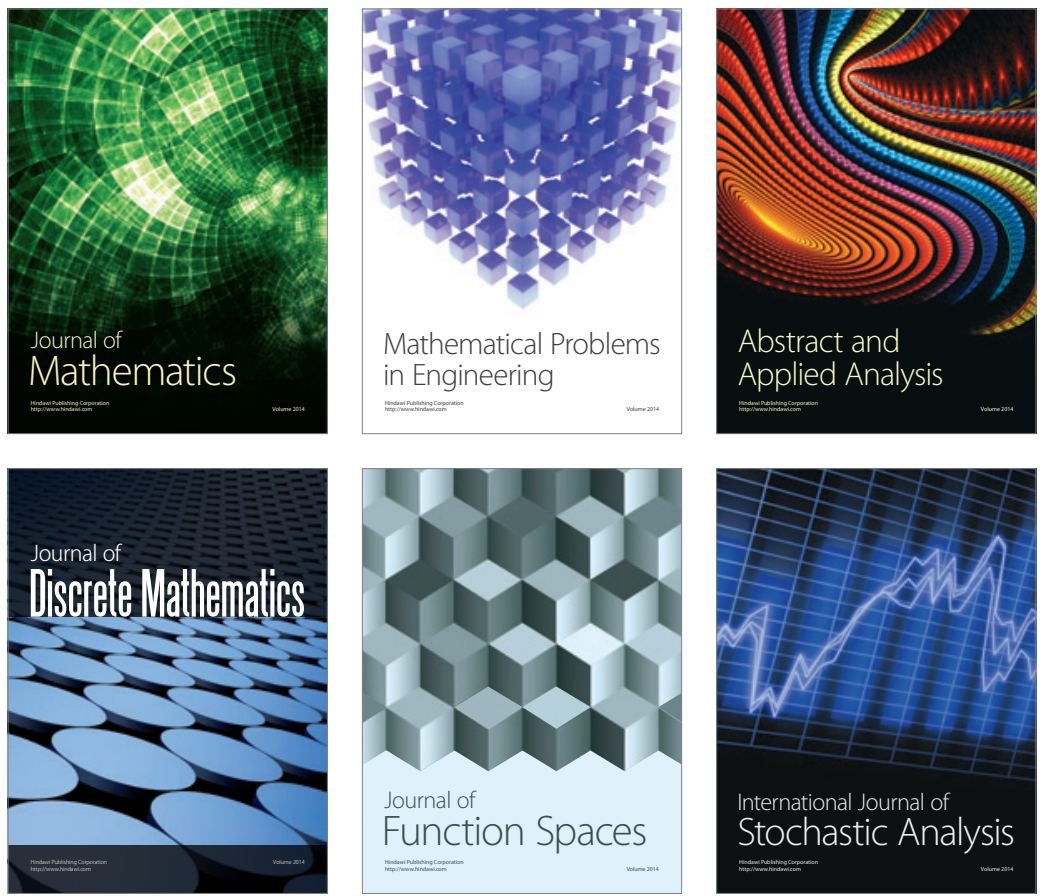

Journal of

Function Spaces

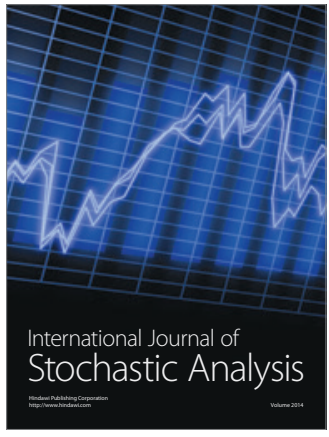

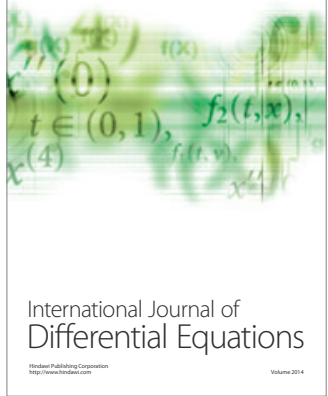
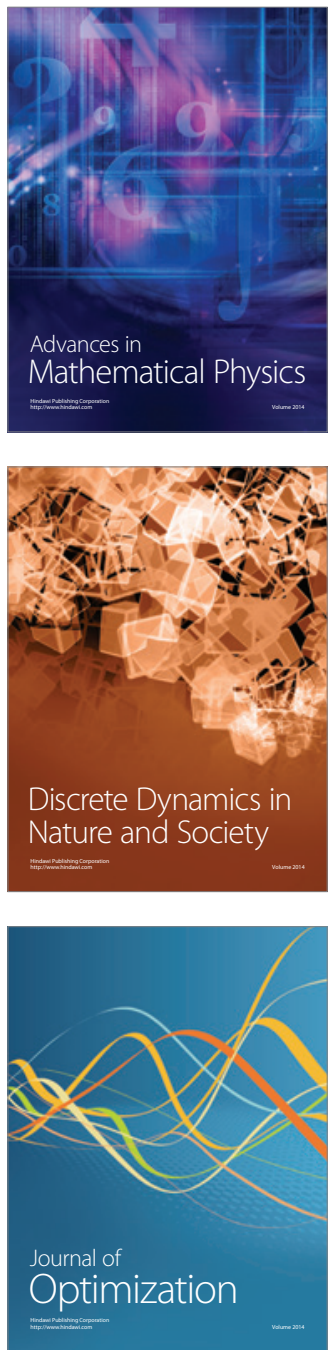